\title{
Controlling Interchain Interactions in Conjugated Polymers: The Effects of Chain Morphology on Exciton-Exciton Annihilation and Aggregation in MEH-PPV Films
}

\author{
Thuc-Quyen Nguyen, ${ }^{\dagger}$ Ignacio B. Martini, ${ }^{\dagger}$ Jei Liu, ${ }^{\ddagger}$ and Benjamin J. Schwartz ${ }^{* \dagger}$ \\ Department of Chemistry and Biochemistry, and Department of Materials Science and Engineering, \\ University of California, Los Angeles, Los Angeles, California 90095-1569
}

Received: September 9, 1999; In Final Form: November 2, 1999

\begin{abstract}
The presence of interchain species in the photophysics of conjugated polymer films has been the subject of a great deal of controversy. In this paper, we present strong evidence that interchain species do form in conjugated polymer films, and that the degree of interchain interactions can be controlled by varying the solvent and polymer concentration of the solution from which the films are cast. Thus, much of the controversy in the literature can be resolved by noting that the polymer samples in different studies had different side groups or were prepared in different ways and thus have different degrees of interchain interaction. The photoluminescence (PL) of poly(2-methoxy-5-(2'-ethylhexyloxy)-1,4-phenylene vinylene), MEH-PPV, changes both its spectral shape and quantum yield when the films are prepared from different solutions or when the morphology is varied by annealing. Increasing the amount of interchain interactions enhances the red portion of the film's PL, a result assigned to a combination of changes in the vibronic structure of the PL of the exciton and increased numbers of weakly emissive interchain species. Photoluminescence excitation spectroscopy shows that excitation to the red edge of the absorption band preferentially enhances the red emission, suggesting that the interchain species are aggregates with a distinct ground state absorption. Scanning force microscopy shows topographic features that correlate with the degree of interchain interactions, verifying that the morphology of conjugated polymer films changes with polymer concentration, choice of solvent, and spin-casting speed. Even at low excitation intensities, photooxidative damage occurs quickly in MEH-PPV films excited in air, and the rate at which damage occurs is sensitive to the packing of the polymer chains. For samples under vacuum at low excitation intensity, a long-lived emissive tail, in combination with excitedstate absorption dynamics that do not match those of the emissive species, provide direct evidence for the production of interchain aggregates. Annealing an MEH-PPV film produces a photophysical signature similar to photooxidation, implying that defects in conjugated polymer films are intrinsic and depend on the details of how the chains are packed. At higher excitation intensities, we find that exciton-exciton annihilation occurs, and that the probability for annihilation can vary by an order of magnitude depending on the degree of interchain contact in the film. Finally, we show that changing the film morphology has a direct effect on the performance of MEH-PPV-based light-emitting diodes. Higher degrees of interchain interaction enhance the mobility of carriers at the expense of lower quantum efficiencies for electroluminescence. Taken together, the results reconcile much of the contradictory literature and provide a prescription for the optimization of conjugated polymer films for particular device applications.
\end{abstract}

\section{Introduction}

Conjugated polymers are a novel class of materials that combine the optical and electronic properties of semiconductors with the processing advantages and mechanical properties of plastics. ${ }^{1}$ When functionalized with flexible side groups, these materials become soluble in common organic solvents and can be solution processed at room temperature into uniform, largearea, optical-quality thin films. ${ }^{2}$ Such films are flexible and easily fabricated into desired shapes that are useful in novel devices. ${ }^{3}$ The ease of polymer processing compared to conventional inorganic semiconductors offers the potential for enormous cost-savings in applications that require visible band-gap semiconductors. Thus, conjugated polymers offer the possibility

\footnotetext{
* Corresponding author e-mail, schwartz@ chem.ucla.edu; fax, (310) 2064038.

Department of Chemistry and Biochemistry.

Department of Materials Science and Engineering.
}

for completely new applications such as cost-effective, largearea luminescent panels or flexible, lightweight displays., ${ }^{4,5}$

Despite this enormous versatility for optoelectronic applications, some of the fundamental physics underlying the construction or optimization of practical devices based on these materials remains controversial or poorly understood. For example, when the chains of a conjugated polymer are isolated in dilute solution, it is well accepted that photoexcitation creates only one electronic species,${ }^{6-9}$ namely, a singlet intrachain exciton. When the polymer chains are in contact in a film, however, estimates of the number of primary photoexcitations that result in interchain species range from essentially zero ${ }^{10}$ to $90 \% .^{11}$ This controversy has ramifications beyond simple differences in interpretation of the photophysics: the formation of weakly emissive interchain species would serve to significantly reduce the efficiency of electroluminescent conjugated polymer devices. If the process that forms these interchain species is intrinsic, then there is no simple relationship between the photolumines- 
cence (PL) and electroluminescence (EL) efficiencies of conjugated polymer films. This leads to the question of whether the polymer-based light-emitting devices already produced have close to the maximum possible efficiency or if there is room for significant improvement.

In this paper, we present evidence that interchain species, which we identify as aggregates, do form in films of conjugated polymers, and that the degree to which they form can be controlled by altering the way in which the chains pack when the films are cast. We will focus our attention on the interchain interactions in phenylene-vinylene (PPV) polymers, in particular poly(2-methoxy-5-(2'-ethylhexyloxy)-1,4-phenylene vinylene), or MEH-PPV (for chemical structure, see inset to Figure 1, below). We find that the degree of interchain interaction affects not only basic photophysical properties such as the shape and position of the PL spectrum and the PL lifetime, but also nonlinear behaviors such as the degree of excitonexciton annihilation. In addition, we demonstrate that the rate at which samples undergo photooxidative damage is also morphology dependent. Thus, we are able to reconcile many of the conflicting results presented in the literature. Finally, we show that the ability to control interchain interactions by altering the film processing directly affects the performance of conjugated polymer-based light-emitting diodes.

Given the extent of conflicting results and interpretation, we spend the remaining sections of the introduction reviewing the types of interchain interactions postulated in conjugated polymers and the controversy over their existence. The rest of this paper is then organized as follows. Section II summarizes our experimental methods, and the results are described in section III. We summarize and conclude in section IV.

A. Aggregates and Excimers in Conjugated Polymers. With all the controversy about interchain species, it is worth exploring the different types of interchain interactions that can occur in conjugated polymers. If the excited state wave function is delocalized over two or more polymer chains, there are several limiting cases to consider. If the excited state delocalization is accompanied by charge separation so the electron resides on one chain and the hole on an adjacent chain, the species is referred to as a "spatially indirect exciton" or "polaron pair". 8,11 On the other hand, if the excited state has no significant degree of charge separation and is shared equally between multiple chains, the species is referred to as an "excimer". ${ }^{12-14}$ A neutral excited state shared between unequal chains (or unequally between similar chains) is sometimes called an "exciplex". ${ }^{15,16}$ There is clearly a continuum of possible interchain excited states between polaron pairs and excimers, and probably all are possible in the inhomogeneous environment of a conjugated polymer film. For any of these interchain states, the delocalization of the excited-state wave function lowers the energy relative to the single-chain exciton, so that interchain luminescence is expected to be red-shifted relative to the standard exciton PL. In addition, the overlap of a delocalized interchain excited state with the single-chain ground-state polymer wave function is expected to be poor, leading to a long radiative lifetime and (due to the presence of nonradiative pathways) a low luminescence quantum efficiency. ${ }^{17-19}$

Samuel and co-workers recently have argued that the formation of emissive excimers is important in the photophysics of a cyano-substituted PPV (CN-PPV). They observed an emission band in the film that was broader, red-shifted, and longer-lived than that in solution, consistent with excimer formation. ${ }^{13,14}$ Although $\mathrm{CN}-\mathrm{PPV}$ is widely regarded as a special case due to the strength of its excimer emission, these authors also noted that $\mathrm{MEH}-\mathrm{PPV}$ is like $\mathrm{CN}-\mathrm{PPV}$ in that it also shows a redshifted and longer-lived PL in films compared to solution. Thus, Samuel and co-workers suggest that interchain excimers also play a role in the excited-state photophysics of MEH-PPV, although not to as large an extent as for $\mathrm{CN}-\mathrm{PPV} .^{13}$

In addition to excimer-like interactions in the excited state, it is possible for conjugated polymers to interact in both the ground and excited states. Delocalization of the wave function over multiple chains in the ground state produces a weak absorption that is red-shifted from that of a single chain. Excitation of the aggregate band typically produces a broad, featureless and highly Stokes-shifted emission. Studies of laddertype poly(para-phenylene), ${ }^{20-22}$ poly(para-pyridyl vinylene), ${ }^{23,24}$ and polyfluorene ${ }^{25}$ deriviatives show strong evidence for aggregate formation in films. Near-field scanning optical microscopy (NSOM) shows that the optical properties of these films vary from location to location, consistent with the idea of spatially localized aggregate species. ${ }^{24,26,27}$ All of these results suggest that conjugated polymer chains can aggregate to form electronic species with ground- and excited-state properties distinct from that of an isolated polymer chain.

We recently have performed a careful study of the photophysics of $\mathrm{MEH}-\mathrm{PPV}$ and have concluded that aggregates play an important role in solution. ${ }^{28}$ Our evidence consists of a new red-shifted absorption band observable via fluorescence excitation that correlates with a weak red-shifted emission. Excitation of the new band produces a long-lived excited-state absorption that does not match the dynamics of the PL, consistent with an interchain species. We found that the degree of aggregation depends on the chemical nature of the solvent and the concentration of polymer in solution. Aggregation is promoted in solvents such as chlorobenzene (CB), where the polymer chains have an open and straight conformation, and is restricted in solvents such as tetrahydrofuran (THF) where the chain tends to form a tight coil. ${ }^{28}$ And as expected, aggregation increases with increasing polymer concentration. Our results are in accord with a growing body of evidence that alkoxy-substituted PPVs undergo aggregation in solution, and that the amount of aggregation depends on changes in the polarity of the solvent, the polymer concentration, and the solution temperature. ${ }^{29-32}$

The distinction between aggregates, excimers and the other varieties of interchain species can be quite hazy. It is well known, for example, that the inhomogeneous environment in a conjugated polymer film leads to excited states that are broadly distributed in energy. Excitation into the inhomogeneous distribution results in rapid energy transfer to the lowest energy states. ${ }^{33-36}$ Thus, the emission in a conjugated polymer can come from a completely different chromophore than the one that was originally excited. Our assignment of the existence of aggregates in solutions of MEH-PPV was predicated on the observation of a distinct ground-state feature, excitation of which led to different photophysics than excitation into the main exciton band. ${ }^{28}$ One can easily imagine, however, that energy transfer can cause intrachain excitons to migrate rapidly to low-energy aggregated sites. The net result would be excitation of a singlechain ground state to produce an aggregated interchain excited state, providing a much more excimer-like behavior. Throughout this paper, we will refer to the interchain species in $\mathrm{MEH}-$ PPV as aggregates, with the caveat that we do not know either the extent of charge transfer or wave function sharing between the chains or the extent to which the interchain species are excited directly from the ground state.

Given that aggregation occurs in many different conjugated polymers and is also evident in solutions of $\mathrm{MEH}-\mathrm{PPV}$, one 
might expect there to be strong evidence for aggregate formation in MEH-PPV films. As pointed out above, however, the existence of interchain species in conjugated polymers has been quite controversial. After exploring the controversy in more detail, we will argue that MEH-PPV aggregates in solution survive the casting process and that the resultant film has a degree of aggregation reminiscent of that in solution.

B. Controversy Over Interchain Interactions in Conjugated Polymers. In a pioneering study, Rothberg and coworkers compared the excited-state photophysics of isolated MEH-PPV chains to those when the chains were in contact in a neat film. ${ }^{8}$ Their femtosecond pump-probe experiments showed strong stimulated emission (SE) with a decay that matched the PL lifetime when the chains were isolated. In the films, however, the experiments revealed only excited state absorption (or PA, photoinduced absorption) at all wavelengths, including those where the polymer luminesces. Furthermore, they found little relationship between the dynamics of the PA and those of the PL. The appearance of an emission-region PA only in films and not in solution suggests a significant fraction of the excited-state species in the film must be interchain; the interchain species have an excited-state absorption spectrum and dynamics distinct from that of the intrachain exciton. ${ }^{8}$ In combination with other experiments studying the excited-state photophysics as the excitation wavelength is varied, Rothberg and co-workers concluded that up to $90 \%$ of the primary photoexcitations in PPV-based polymer films are interchain. ${ }^{11,37}$

In contrast, work from several groups has argued that stimulated emission does exist in films of conjugated polymers and that there is little evidence for interchain species in phenylene-vinylene polymers. First, Greenham et al. did a series of careful PL quantum yield measurements on films of several PPV derivatives using an integrating sphere. ${ }^{10}$ They found a consistent relationship between the radiative lifetime, PL quantum yield and PL decay rate, leaving no room for significant branching to an interchain species. Soon thereafter, Frolov et al. demonstrated the formation of an "excitonic switch" in an alkoxy-substituted PPV similar to MEH-PPV. ${ }^{38}$ In this work, a strong "dump" pulse applied to the center of the SE band causes a reduction in the intensity of the PA in the nearinfrared. This result shows that the SE and PA bands are correlated: removal of emissive excitons by SE proportionally removes the species responsible for the excited-state absorption, suggesting that none of the excited-state absorption is the result of interchain species. Finally, Vacar et al. reexamined the excitation wavelength dependence of the photophysics of MEH-PPV films and found that the excited-state properties were independent of pump wavelength, ${ }^{39}$ providing no evidence for interchain species.

McBranch and co-workers have attempted to reconcile these contradictory results by studying the intensity dependence of the excited-state transients in films of an MEH-PPV oligomer. ${ }^{40-42}$ At low excitation intensities, these workers found identical SE and PA dynamics, suggesting that only a single, intrachain species is needed to explain the photophysics. At higher intensities, a new PA band appeared whose magnitude increased quadratically with intensity. The growth of the new PA band also correlated with a fast decay of the SE. These results suggest that interchain species result from an interaction between excitons. Thus, interchain species are produced at high excitation intensity either from doubly excited chain segments or by exciton-exciton (bimolecular) recombination. ${ }^{40-42}$ Denton et al. recently have performed similar experiments on PPV films in combination with photoconductivity measurements, and claim that the products of exciton-exciton annihilation are absorbing charge carriers. ${ }^{43}$ In more recent work, McBranch et al. found that the PA band associated with the interchain species in conjugated polymer films appears linearly rather than nonlinearly with intensity, indicating that interchain species formation in conjugated polymer films may possibly be mediated by defects. ${ }^{44}$ Similar intensity-dependent results using MEH-PPV films also have been obtained recently by Dogariu et al. ${ }^{45}$ Because the original pump-probe experiments of Rothberg and co-workers ${ }^{8}$ were performed at high excitation intensity and the studies of Greenham et al., ${ }^{10}$ Frolov et al., ${ }^{38}$ and Vacar et al. ${ }^{39}$ were performed at lower excitation intensities, the possibility of intensity-dependent production of an interchain species could explain the observed discrepancies.

Although the possibility of intensity-dependent interchain species can explain most of the aforementioned contradictory results, Rothberg and co-workers have performed new experiments which suggest that interchain species are prevalent in conjugated polymers even at low excitation intensities. ${ }^{46,47}$ Using time-correlated single-photon counting, these workers found evidence for a long-lived, red-shifted emission in $\mathrm{MEH}-\mathrm{PPV}$ films at liquid nitrogen temperatures that is distinct from the intrachain PL. In agreement with Samuel et al., ${ }^{13}$ they assigned this emission to interchain excimers and noted that the long radiative lifetime of the interchain species would cause its emission to be effectively quenched at room temperature. By comparing the relative amplitudes of the intra and interchain luminescence, Rothberg and co-workers conclude that the quantum yield for the formation of interchain species in $\mathrm{MEH}-$ PPV is near $50 \% .46,47$

All of these data still leave open the question of the role of interchain species in films of MEH-PPV. It is clear that the photophysics at high excitation intensities are much more complex in conjugated polymer films than in solution. On the other hand, the existence of an intensity-dependent interchain species does not preclude the possibility of intrinsic interchain species at low intensity. After reviewing previous work on the high-intensity photophysics of these materials, we will argue that the intensity-dependence is dependent on the degree of aggregation and morphology of the conjugated polymer film.

C. Exciton-Exciton Annihilation, Line Narrowing, and Photochemical Damage. At high excitation densities $\left(\geq 10^{19}\right.$ $\mathrm{cm}^{-3}$ ), the emission quantum yield from phenylene-vinylene polymer films has been observed to drop. ${ }^{48}$ This decrease in quantum yield, assigned to exciton-exciton annihilation (EEA), can be severe: the quantum yield drops by over half for a 1 order of magnitude increase in the excitation intensity. Indeed, once out of the linear regime, the magnitude of polymer fluorescence appears to scale as the square root of the input intensity, a result consistent with bimolecular recombination. ${ }^{43,49}$ Fits to simple kinetic models that include a bimolecular recombination term are consistent with the excitation intensity dependence of both the decay time of the luminescence and the loss in quantum yield using a single set of parameters. ${ }^{48,49}$ Similar results have also been obtained for polyfluorene copolymers. ${ }^{50}$ The species produced following the biexcitonic interaction is what McBranch and co-workers ${ }^{40-42}$ and Denton et al. ${ }^{43}$ believe to be responsible for the intensitydependent PA.

Another phenomenon in conjugated polymer films that takes place at the same excitation intensities as E-EA is line narrowing ${ }^{51-62}$ or optically pumped lasing. ${ }^{63-68}$ It is difficult to believe, however, that both E-EA and line narrowing can take place simultaneously; the mechanism which produces line 
narrowing is expected to increase, not decrease, the emission quantum yield. ${ }^{69-72}$ Either a decaying exciton stimulates emission from its neighbors before non-radiative mechanisms can quench the luminescence, or E-EA rapidly lowers the exciton density to the point where stimulated emission cannot occur. We recently have argued that some of the reported drops in quantum yield at high excitation intensity might be due to the anisotropic nature of SE resulting from the natural planar waveguide formed by conjugated polymer films. ${ }^{73}$ This would lead to different PL quantum yields in different spatial directions, possibly masquerading as a drop in overall quantum efficiency. This also might explain the fact that the bimolecular recombination coefficients reported in emission quenching studies for PPV vary by over an order of magnitude. ${ }^{43,48,49,71}$

In addition to line narrowing and E-EA, there are also reports of photochemical damage to conjugated polymers at high excitation intensities. Many studies have reported a link between photooxidation and reduced PL quantum yield. ${ }^{74-76}$ Several groups also have argued that irreversible photodamage can occur in conjugated polymer samples at relatively low $\left(\leq 10^{18} \mathrm{~cm}^{-3}\right)$ excitation intensities, ${ }^{39,49}$ even in the vacuum of a cryostat. ${ }^{40}$ The chief signature of photochemical damage is a rapid decay of SE and the appearance of PA in the emission region; the PA in the near-infrared is only slightly affected. This type of result suggests that the observation of only PA in the emission region in the original MEH-PPV film experiments of Rothberg and co-workers ${ }^{8}$ is the result of a photodegraded sample rather than the presence of large numbers of interchain species.

There is yet one more peculiarity concerning the highintensity photophysics of $\mathrm{MEH}-\mathrm{PPV}$ : line narrowing is readily observed when the films are cast from THF, but not when the films are cast from $\mathrm{CB} .{ }^{51}$ Variations of the line-narrowing behavior and stimulated emission cross-section with film preparation conditions have also been observed for other conjugated polymers. ${ }^{54,55,72}$ This suggests that changing the film morphology by casting the polymer films from different solvents alters the interchain interactions in the films. Different degrees of interchain interaction could change the line-narrowing photophysics by introduction of a new interchain PA that lowers the effective gain, alteration of the rate of E-EA, or modification of the threshold for photochemical damage. It is exactly this kind of dependence of the excited state photophysics on film morphology that is the subject of this paper. We will argue below that the packing of the chains in the film critically changes the cross-section for bimolecular recombination. When the chains are relatively isolated, the probability for E-EA is small and line narrowing can occur. When the chains are more highly aggregated, E-EA occurs readily and the exciton density is reduced quickly enough that line narrowing cannot occur. We also note that the original experiments of Rothberg and co-workers were performed on $\mathrm{MEH}-\mathrm{PPV}$ films cast from $\mathrm{CB},{ }^{8}$ but that their subsequent work ${ }^{46,47}$ as well as that of Vacar et al. ${ }^{39}$ and Dogariu et al. ${ }^{45}$ was performed on MEH-PPV films cast from THF. Thus, we will attempt to reconcile many of the contradictory results in the literature by noting that even for pristine conjugated polymers, the morphology of the film changes both the photophysics and the susceptibility to photochemical damage.

\section{Experimental Section}

The MEH-PPV used in all of the experiments performed here was synthesized following the standard literature procedure. ${ }^{77,78}$ For all of the experiments listed below, we found qualitatively similar results for material produced from two different synthetic batches. The molecular weight of the polymer in each batch was determined by gel permeation chromatography (GPC) to be $535000 \mathrm{~g} / \mathrm{mol}$ and $1000000 \mathrm{~g} / \mathrm{mol}$, respectively. All sample storage, handling and processing was carried out in the inert environment of a nitrogen drybox. Sample solutions in $\mathrm{CB}$ or $\mathrm{THF}$ were prepared by dissolving the appropriate amount of polymer into the desired solvent and stirring for several hours in the dark. Solutions were spin-coated onto glass substrates immediately prior to use. Spin-coated films were heated at $50{ }^{\circ} \mathrm{C}$ for several hours to ensure removal of the solvent. All of the experiments reported here were performed at room temperature. Most of the films used in this study had optical densities near the absorption maximum greater than 3 , making it difficult to collect reliable UV-visible absorption data. The PL and photoluminescence excitation (PLE) spectra were measured on a Fluorolog-3 (Instrument S. A. \& Co.) using a xenon lamp for the excitation source. To minimize the effects of self-absorption from the optically dense samples, PL was collected from the front face of the MEH-PPV film samples. Although the measurement of absolute quantum yields from solid samples can be difficult due to sample geometry and waveguide effects, ${ }^{10,73}$ approximate relative fluorescence quantum yields were obtained by comparing the frequency-cubed weighted and integrated PL spectra of the different samples.

Differential scanning calorimetry (DSC) scans were taken with a Perkin-Elmer Pyris-1 instrument using indium and zinc as standards under nitrogen flow. The heating and cooling rates were $15^{\circ} \mathrm{C} / \mathrm{min}$. The glass transition temperature, $T_{\mathrm{g}}$, of the $\mathrm{MEH}-\mathrm{PPV}$ used in our experiments was determined by DSC to be $216 \pm 10^{\circ} \mathrm{C}$. Annealed MEH-PPV films were prepared by heating previously spin-cast films to $215^{\circ} \mathrm{C}$ in the nitrogen drybox for several hours.

Topographic images of the MEH-PPV film samples were obtained using a scanning force microscope (Park Scientific) with $5 \mu \mathrm{m}$ scanners. The scanning conditions were similar to those of previous work on polysiloxane monolayers. ${ }^{79}$ Microfabricated triangular $\mathrm{Si}_{3} \mathrm{~N}_{4}$ cantilevers (Park Scientific) with a normal spring constant of $0.05 \mathrm{~N} \mathrm{~m}^{-1}$ were used for the measurements. The experiments were done in air under ambient conditions in the constant-force mode, with an applied loading force of $1 \mathrm{nN}$. The scanning frequencies used were between 0.5 and $1 \mathrm{~Hz}$.

Time-resolved SE and PA measurements were made using a regeneratively amplified Ti:sapphire laser which produces $\sim 120$ fs light pulses centered at $800 \mathrm{~nm}$ with $1 \mathrm{~mJ}$ of energy at a 1 $\mathrm{kHz}$ repetition rate (Spectra Physics). These pulses pump a dualpass optical parametric amplifier (OPA). For stimulated emission (SE) experiments, the OPA is set to produce signal and idler beams at 1250 and $2220 \mathrm{~nm}$, respectively. The $1250 \mathrm{~nm}$ signal beam propagates collinearly with the residual $800 \mathrm{~nm}$ light into a type II BBO crystal to produce femtosecond pulses at 485 $\mathrm{nm}$ for excitation of MEH-PPV by sum-frequency mixing (SFM). The probe light at $625 \mathrm{~nm}$ is produced by second harmonic generation $(\mathrm{SHG})$ of the residual $1250 \mathrm{~nm}$ light from the SFM process in a second BBO crystal. Additional SE experiments were performed with a $590 \mathrm{~nm}$ probe beam generated by SFM in a type I BBO crystal using the $2220 \mathrm{~nm}$ idler beam and the residual $800 \mathrm{~nm}$ light from the first SFM process. For photoinduced absorption (PA) experiments, the second crystal (SHG or SFM) was removed and filters were used to select only the residual $800 \mathrm{~nm}$ light for use as the probe. Samples were pumped through the transparent substrate to minimize simultaneous exposure to light and oxygen. As discussed below, we found reproducible signals when exciting 
this way at low intensities for samples under vacuum in a cryostat. The pump and probe beams are focused collinearly in the sample, and wave plates and polarizers are used to orient the relative polarization of the pump and probe beams to the magic angle $\left(54.7^{\circ}\right)$ to prevent interference from dynamical processes (such as energy transfer ${ }^{33-36}$ ) that can reorient the transition dipole of the species being probed. ${ }^{80}$

For all of the femtosecond experiments, a small part of the probe light is split off to serve as a reference, and the remainder is attenuated with neutral density filters to serve as the probe pulse. The signal and reference beams are then collected on $\mathrm{Si}$ photodiodes whose output is digitized on every laser shot using a fast gated current-integrating 11-bit analog-to-digital converter (A/D) (LeCroy 4300B). The A/D and computer are fast enough to allow on-the-fly rejection of any laser shots with intensities outside preset bounds; typically we reject any pulses whose intensity deviates more than $\pm 15 \%$ from the average. The experimental signal is then normalized in two ways. First, the digital values for the signal and reference beams are divided on each shot, normalizing away fluctuations in the probe intensity. Second, small baseline drifts and pump intensity fluctuations are accounted for by mechanically chopping the pump beam, and subtracting the ratio of the digitized signal and reference beams with the pump off from that of the adjacent shot with the pump on. ${ }^{81}$ The time delay between the pump and probe pulses is varied mechanically by a computercontrolled delay stage with $0.5 \mu \mathrm{m}$ resolution. Typical baseline noise when averaging 300 shots $(0.3 \mathrm{~s})$ per stage position is a change in absorbance of $\sim 2 \times 10^{-4}$. This easily allows us to collect $\sim 1 \mathrm{mOD}$ transients resulting from excitation fluences of $\sim 15 \mu \mathrm{J} / \mathrm{cm}^{2}$ (excitation densities $\sim 10^{18} \mathrm{~cm}^{-3}$ ) with good signal-to-noise ratios using only a single pass of the delay stage: a typical scan with 100 delay positions can be collected in roughly $1 \mathrm{~min}$. As discussed below, this ability to rapidly scan with such small signal levels is critical to determining the role of photochemical damage in the MEH-PPV films.

The excitation energy at the sample is calculated using the measured intensity of the pump beam on a photodiode and the known transmission of calibrated neutral density filters. The excitation spot size at the sample was determined to be $\sim 250$ $\mu \mathrm{m}$ by measuring the transmission of the pump beam through a series of calibrated pinholes placed at the sample position. Due to the uncertainties in the measurements of both the spot size and the energy at low intensities, the absolute values for the pump fluence reported below are likely accurate only to $\pm 50 \%$. Excitation densities were determined using the calculated pump fluence and the fraction of pump light absorbed by the sample over its thickness. For the $485 \mathrm{~nm}$ pump photons used here, we calculate that an excitation fluence of $1 \mu \mathrm{J} / \mathrm{cm}^{2}$ produces an excitation density of $\sim 8 \times 10^{16} \mathrm{~cm}^{-3}$. Due to the combination of errors inherent in measuring the absorption depth of the MEH-PPV films and those in determining the pump fluence, we believe our calculated absolute excitation densities (and hence bimolecular recombination coefficients) are accurate only within a factor of 2 . As discussed below, the relative quantum yield of excitons produced following excitation is not the same for different films, leading to an additional undetermined scaling factor in computing the excitation density. The relative pump fluences and excitation densities on different scans for the same film, however, should be accurate to within a few percent since the relative change in pump pulse energy from scan to scan can be measured quite accurately.

Two sets of polymer light-emitting diodes (LEDs) were constructed utilizing $\mathrm{MEH}-\mathrm{PPV}$ films cast from either $\mathrm{CB}$ or
THF solutions as the active layer. Both sets of devices were fabricated in an inert atmosphere using precleaned indiumtin-oxide (ITO) substrates as the anode. In one set of diodes, the conducting polymer 3,4-polyethylenedioxythiophene-polystyrenesulfonate (PEDOT) was spin-coated onto the ITO substrate and subsequently baked at $120{ }^{\circ} \mathrm{C}$ for $2 \mathrm{~h}$ to serve as a hole transport layer. The active $\mathrm{MEH}-\mathrm{PPV}$ luminescent layer was then obtained by spin casting from a $1 \%$ weight/volume (w/v) solution in $\mathrm{CB}$ or THF and then baked at $70^{\circ} \mathrm{C}$ for $2 \mathrm{~h}$. In the second set of diodes, the $\mathrm{MEH}-\mathrm{PPV}$ layers were spun directly onto the precleaned ITO substrates. Spin speeds were chosen to ensure that the thickness of the MEH-PPV layer in all of the devices was about $1200 \AA$ as measured with an alphastep profilometer. For both sets of devices (with and without PEDOT), metal cathodes consisting of calcium (500 $\AA$ ) with an additional encapsulating layer of aluminum (1000 ̊) were thermally evaporated at $\sim 10^{-6}$ Torr. The total light-emitting area is about $0.12 \mathrm{~cm}^{2}$. The current-brightness-voltage measurements ( $\mathrm{I}-\mathrm{V}$ and $\mathrm{L}-\mathrm{V}$ curves) were carried out in a nitrogen environment using a HP 4155B Semiconductor Parameter Analyzer. Relative device efficiencies (photons/electron) were determined by dividing the measured brightness (voltage on the detection photodiode) by the current passing through the device.

\section{Results and Discussion}

Our operative definition of a conjugated polymer aggregate is a place where two or more chain segments come together and share their $\pi$-electron density. Our definition of such aggregates is photophysical; the extent of $\pi$-electron delocalization must be enough to significantly alter the wave function from that of a single-chain chromophore. Thus, we expect that aggregated chains have $\pi$-electron contact for approximately the length of the exciton $\left(\sim 4\right.$ repeat units $\left.{ }^{82}\right)$. With this definition, strongly tangled chain segments which cross at 90 degrees are not considered aggregated: they would have minimal $\pi$-electron overlap and thus no distinct photophysical signature. We also note that our definition of an aggregate does not require that the shared chromophores come from physically distinct chains: one chain wrapped around on itself can form an aggregate if two of its chromophores share enough $\pi$-electron density. When we use the words "interchain interactions" in this paper, we imply that $\pi$-electron density is shared between multiple chromophores that can come from either the same or different polymer chains.

In our previous work, we argued that the extent of aggregation of MEH-PPV in solution could be controlled by the choice of solvent and concentration. ${ }^{28}$ Aromatic solvents such as CB have a preferential interaction with the aromatic backbone of the polymer chain, and thus the chains adopt a rigid, open conformation in solution. With the backbone open and exposed, it is straightforward for the $\pi$-electrons on one chain to overlap with those on a neighboring chain. Increasing the concentration offers a greater number of segments available for interaction, as a result, the extent of aggregation is increased. Nonaromatic solvents such as THF, on the other hand, have a preferential interaction with the polymer's side groups. Thus, the polymer chains in THF coil tightly to maximize solvent-side group interactions and minimize exposure of the aromatic backbone to the solvent. Evidently, the MEH-PPV chains do not coil in THF in a manner that allows significant $\pi$-electron interactions between chromophores on the same chain. Aggregation can still occur in THF, but only at high enough concentrations to force adjacent polymer coils together so that $\pi$-electron density is shared over a sufficient contiguous distance. ${ }^{28}$ 


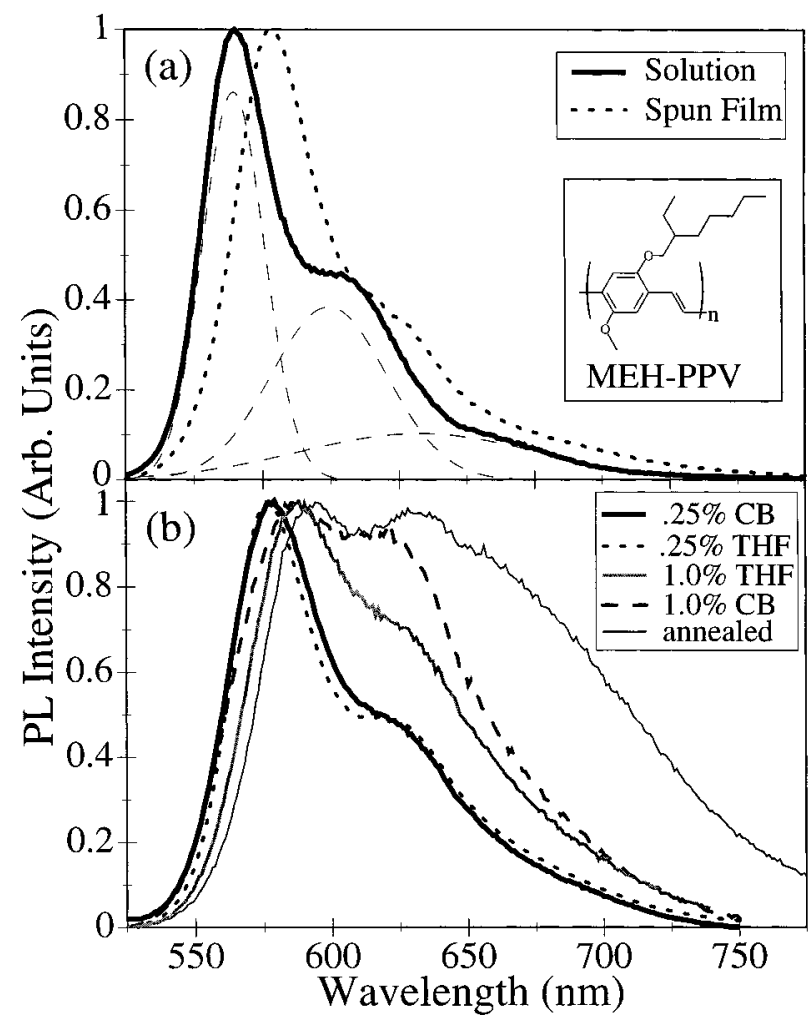

Figure 1. Normalized photoluminescence (PL) spectra of MEH-PPV in different environments. (a) PL of a $0.25 \%$ w/v solution of MEHPPV in CB (solid curve), and the film resulting from spin-casting the solution (dotted curve). The small dashed curves show Gaussian fits to the three visible peaks of the solution PL; fit parameters for the film PL are summarized in Table 1. (b) PL of MEH-PPV films cast from a $0.25 \% \mathrm{w} / \mathrm{v}$ solution in CB (solid curve, same as dashed curve in (a)), a $0.25 \% \mathrm{w} / \mathrm{v}$ solution in THF (dotted curve), a $1.0 \%$ solution in THF (gray solid curve), a 1.0\% solution in CB (dashed curve) and the film cast from the $1.0 \% \mathrm{CB}$ solution after annealing (thin solid curve). The inset shows the chemical structure of MEH-PPV.

The purpose of this paper is to test the hypothesis that the degree of aggregation in solution is preserved through the casting process and survives into the film. If true, this idea suggests that aggregation is indeed important in conjugated polymers since most films are spin-coated from solutions with high enough concentration that aggregates are prevalent. This hypothesis also suggests that the degree of aggregation in the film can be tailored by changing the properties of the solution from which the film is cast, making it possible to optimize the interchain interactions in conjugated polymer films for particular applications.

In the remainder of this section, we present experimental evidence that interchain interactions between conjugated polymers can indeed be controlled by the way in which the films are prepared. We will focus our attention on MEH-PPV films cast from 4 different solutions: high concentration $(1.0 \% \mathrm{w} / \mathrm{v})$ in $\mathrm{CB}$; high concentration $(1.0 \% \mathrm{w} / \mathrm{v})$ in THF, low concentration $(0.25 \% \mathrm{w} / \mathrm{v})$ in $\mathrm{CB}$, and low concentration $(0.25 \% \mathrm{w} / \mathrm{v})$ in THF. It is worth noting that preliminary evidence suggests that the spin speed also affects the morphology and photophysics of conjugated polymer films; we will explore this in detail in future work. ${ }^{83}$ We also varied the film morphology by annealing: heating the films to $T_{\mathrm{g}}$ for several hours allows the chains to reorganize and produce lower energy structures, presumably increasing the amount of interchain interactions.

A. Morphology Effects on the PL of MEH-PPV Films. Figure 1(a) shows the PL spectrum of a $0.25 \%$ w/v solution of
TABLE 1: Fit Parameters ${ }^{a}$ to Normalized PL Spectra of the MEH-PPV Films Presented in Figure 1 and PL Quantum Yields $^{b}$

\begin{tabular}{clllc}
\hline $\begin{array}{c}\text { MEH-PPV film } \\
\text { cast from: }\end{array}$ & peak 1 & peak 2 & peak 3 & $\begin{array}{c}\text { PL quantum } \\
\text { yield }^{b}\end{array}$ \\
\hline $0.25 \%$ CB soln & $\lambda_{1}=564$ & $\lambda_{2}=599$ & $\lambda_{3}=632$ & N/A $^{b}$ \\
& $\sigma_{1}=11.3$ & $\sigma_{2}=21.2$ & $\sigma_{3}=44.0$ & \\
& $a_{1}=0.86$ & $a_{2}=0.38$ & $a_{3}=0.10$ & \\
$0.25 \%$ w/v THF & $\lambda_{1}=575$ & $\lambda_{2}=613$ & $\lambda_{3}=650$ & 0.32 \\
& $\sigma_{1}=13.0$ & $\sigma_{2}=25.3$ & $\sigma_{3}=43.6$ & \\
& $a_{1}=0.83$ & $a_{2}=0.37$ & $a_{3}=0.17$ & \\
$0.25 \%$ w/v CB & $\lambda_{1}=576$ & $\lambda_{2}=615$ & $\lambda_{3}=633$ & 0.31 \\
& $\sigma_{1}=14.6$ & $\sigma_{2}=21.6$ & $\sigma_{3}=44.5$ & \\
$1.0 \%$ w/v THF & $a_{1}=0.83$ & $a_{2}=0.28$ & $a_{3}=0.22$ & \\
& $\lambda_{1}=583$ & $\lambda_{2}=618$ & $\lambda_{3}=652$ & 0.17 \\
& $\sigma_{1}=15.1$ & $\sigma_{2}=24.6$ & $\sigma_{3}=44.5$ & \\
$1.0 \%$ w/v CB & $a_{1}=0.70$ & $a_{2}=0.48$ & $a_{3}=0.29$ & \\
& $\lambda_{1}=578$ & $\lambda_{2}=619$ & $\lambda_{3}=654$ & 0.12 \\
& $\sigma_{1}=17.0$ & $\sigma_{2}=22.6$ & $\sigma_{3}=39.3$ & \\
$1 \% \mathrm{CB}$, annealed & $a_{1}=0.75$ & $a_{2}=0.65$ & $a_{3}=0.35$ & \\
& $\lambda_{1}=587$ & $\lambda_{2}=620$ & $\lambda_{3}=671$ & 0.07 \\
& $\sigma_{1}=13.3$ & $\sigma_{2}=29.7$ & $\sigma_{3}=54.5$ & \\
& $a_{1}=0.49$ & $a_{2}=0.48$ & $a_{3}=0.67$ &
\end{tabular}

${ }^{a}$ Each PL spectrum is fit to:

$$
\sum_{i=1}^{3} a_{\mathrm{i}} \exp \left[-\frac{1}{2}\left(\frac{x-\lambda_{\mathrm{i}}}{\sigma_{\mathrm{i}}}\right)^{2}\right]
$$

${ }^{b}$ Only relative quantum yields were determined experimentally; absolute numbers were obtained by assigning the quantum yield of the film cast from the $1.0 \% \mathrm{w} / \mathrm{v} \mathrm{CB}$ solution to $12 \%$, see text. The relative PL quantum yield of the solution cannot be compared directly to those of the films because of differences in the sample geometry. ${ }^{c}$ Fit parameters for $\mathrm{MEH}-\mathrm{PPV}$ in solution, not the film cast from the solution.

$\mathrm{MEH}-\mathrm{PPV}$ in $\mathrm{CB}$ (heavy solid curve) and the film which resulted from spin-casting this solution onto a glass substrate (dotted curve). The PL spectra have been normalized to the same maximum value for better comparison and both show three discernible peaks. The thin dashed curve shows three Gaussians whose sum is fit to the solution PL. The overall fit is indistinguishable from the data on the scale of the figure; the Gaussian fit parameters for all of the PL spectra shown in Figure 1 are summarized in Table 1.

The three peaks in the CB solution PL spectrum (Figure 1(a), heavy solid curve) are roughly equally spaced in energy. Thus, the logical assignment is that the entire emission comes from a single electronic state and that the peaks represent vibronic structure (the $0-0,0-1$, and $0-2$ emission bands) from a mode that is strongly displaced upon excitation. The observed splitting corresponds well to the energy of a $\mathrm{C}=\mathrm{C}$ stretch, a motion that is expected to be strongly displaced when the benzoid-like polymer ground state is excited to the quinoid-like excited state. In addition to the emission consisting of vibronic structure, we noted in previous work that MEH-PPV aggregates in solution have a weak red emission that peaks near $660 \mathrm{~nm} .{ }^{28}$ This means that aggregate species in solution fluoresce in roughly the same place as the $0-2$ emission band. The magnitude of the aggregate emission in low concentration solutions, however, is quite low. ${ }^{28}$ Thus, we assign the features in the solution PL spectrum to vibronic structure, with the possibility of a very small contribution from aggregate emission in the red tail near the 0-2 band.

The MEH-PPV film cast from the $0.25 \% \mathrm{w} / \mathrm{v}$ solution has a PL spectrum that is noticeably red-shifted from that in solution (dotted curve, Figure 1(a)), consistent with the "gas-to-crystal" effect. $^{84}$ This effect results from the fact that the dipole of an MEH-PPV excited state interacts more strongly with its 


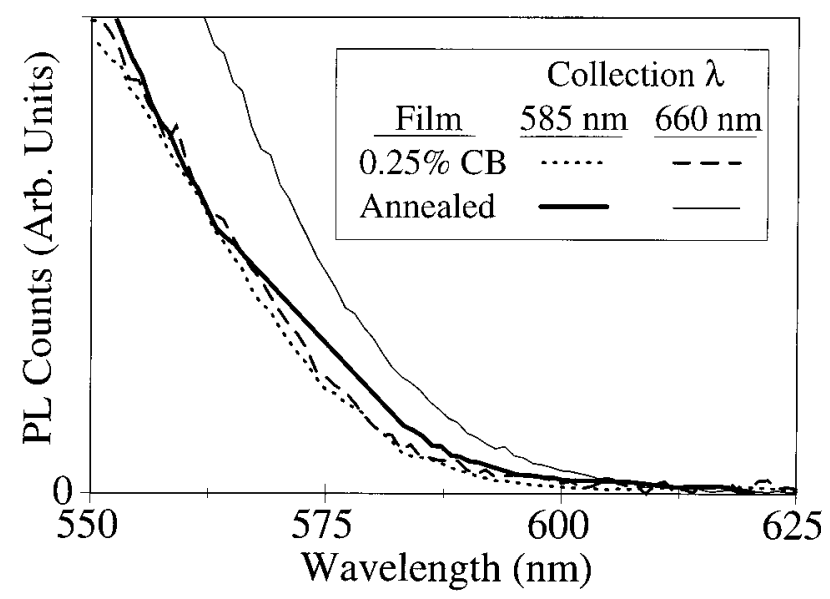

Figure 2. Photoluminescence excitation (PLE) spectra for emission collected at $585 \mathrm{~nm}$ for an MEH-PPV film cast from $0.25 \% \mathrm{w} / \mathrm{v} \mathrm{CB}$ solution (dotted curve) and an MEH-PPV film cast from $1.0 \% \mathrm{w} / \mathrm{v}$ CB solution after annealing (heavy solid curve). The PLE spectra for emission collected at $660 \mathrm{~nm}$ for the $0.25 \%$ CB-cast film (dashed curve) and for the annealed film (thin solid curve) are also shown.

surroundings in the highly polarizable environment of a conjugated polymer film than in solution. The stronger polarization interaction with the environment leads to a red-shifted emission. Other than the red-shift, the film PL retains the same general shape as in solution: all three bands shift by roughly the same amount with little change in the relative peak heights or widths. Unlike our previous work on high concentration solutions, ${ }^{28}$ red-edge excitation of this film shows no enhancement of the red emission near the $0-2$ band. This is shown explicitly by the photoluminescence excitation (PLE) spectra in Figure 2. Collection of the PL at $585 \mathrm{~nm}$ (dotted curve), the peak of the $0-0$ band where aggregates are not expected to emit, ${ }^{28,46,47}$ results in an excitation profile that matches the UVvisible absorption spectrum. Collection of the PL at $660 \mathrm{~nm}$ (dashed curve), near the expected peak of the aggregate emission, produces an excitation profile essentially identical to that at $585 \mathrm{~nm}$, providing little evidence for aggregate emission. The quantum yield for aggregate emission should be much smaller in films than in solution at room temperature; the aggregate film quantum efficiency should be on the order of only a few percent. ${ }^{46,47}$ Since the PL quantum efficiency of the exciton is expected to be on the order of tens of percent, ${ }^{10}$ the ability to distinguish the aggregate and exciton by PLE in films requires a large fraction of the species produced upon excitation to be aggregates. Therefore, the PLE data indicate that relatively few of the excitations in the MEH-PPV film cast from the $0.25 \% \mathrm{w} / \mathrm{v}$ solutions result in emission from aggregates.

Figure 1(b) compares the normalized PL of MEH-PPV films cast from four different solutions: $0.25 \% \mathrm{w} / \mathrm{v}$ in $\mathrm{CB}$ (heavy solid curve), $0.25 \% \mathrm{w} / \mathrm{v}$ in THF (dotted curve), $1.0 \% \mathrm{w} / \mathrm{v}$ in THF (solid gray curve), and $1.0 \% \mathrm{w} / \mathrm{v}$ in $\mathrm{CB}$ (dashed curve). The PL from the films cast from both low concentration solutions are similar, but the shape of the PL from the films cast from the $1.0 \% \mathrm{w} / \mathrm{v}$ solutions changes with solvent. The heights of the three emission peaks also change with the solution concentration, with the redder peaks increasing in intensity relative to the bluest $(0-0)$ peak. The red-shift and increase in relative height of the two reddest peaks are accentuated in the PL of the annealed film, which is shown as the thin solid curve. Annealing any of the MEH-PPV films leads to a qualitatively similar PL, so only the PL of the $1.0 \%$ CB-cast annealed film is shown as representative. Excitation spectra of the annealed film for PL collected at $590 \mathrm{~nm}$ (heavy solid curve) and 660 nm (thin solid curve) are also shown in Figure 2. As expected, the 590-nm PLE spectrum of the annealed film is identical to the absorption spectrum. Unlike what was observed for the $0.25 \%$ CB-cast film, however, the 660-nm PLE of the annealed film shows a clear signature of red-edge excitation leading to enhancement of the red emission. In accord with our previous work on $\mathrm{MEH}-\mathrm{PPV}$ in solution, ${ }^{28}$ we assign the weakly absorbing, weakly emitting red-shifted species to aggregates. The 660-nm PLE spectra of the MEH-PPV films cast from the $1.0 \% \mathrm{w} / \mathrm{v}$ solutions (not shown) show a rising edge between that of the $0.25 \%$ solution-cast films and the annealed films. Given the low aggregate emission quantum yield in films, the detection of aggregate emission by PLE implies that large fractions of the excited species in the $1 \%$ solution-cast films (and even larger fractions in the annealed films) are aggregates.

We have already argued that the degree of aggregation of $\mathrm{MEH}-\mathrm{PPV}$ in solution is greater in CB than in THF and is larger at higher concentrations. ${ }^{28}$ Our working hypothesis is that memory of the interchain interactions in solution is preserved through the casting process and survives into the film. Thus, the red-shift of the PL and increase in the height of the two red peaks relative to the $0-0$ band must be a direct reflection of increased interchain interactions in the film. The PL and PLE of the annealed film are also consistent with this idea. Annealing produces a film where the chains have had the opportunity to pack into low energy structures with a high degree of interchain contact. The main question that remains to be answered is why does increasing the amount of interchain interactions change the relative heights of all three peaks instead of just the reddest peak? We see two possible answers to this question: (1) that the emission from the aggregate, which peaks near $660 \mathrm{~nm}$, has enough amplitude near the $0-1$ band at $625 \mathrm{~nm}$ to change the relative heights of both of the red peaks relative to the $0-0$ band, or (2) that the emission of the intrachain exciton changes its vibronic structure in different film environments.

The possibility that a fluorescent interchain species in $\mathrm{MEH}-$ PPV films can change the shape of the PL already has been postulated by groups working on MEH-PPV-based LEDs that emit different colors when the films are prepared in different ways. ${ }^{85,86}$ The PLE results in Figure 2 argue that the emission at the position of the $0-2$ band near $660 \mathrm{~nm}$ in the annealed films and in the films cast from high concentration solutions is due in part to aggregates. Thus, the relative increase in the height of this band can be explained by the facts that increasing the concentration of the solution increases aggregation and that changing the solvent to $\mathrm{CB}$ from THF promotes aggregation. ${ }^{28}$ The emission that does come from aggregates in MEH-PPV, however, peaks near $660 \mathrm{~nm}$ and has a smaller contribution at the position of the $0-1$ band near $625 \mathrm{~nm} \cdot{ }^{28,46,47}$ The Gaussian fit parameters in Table 1 show that the increase in amplitude of the $660-\mathrm{nm}$ band with increasing interchain interactions, which could reasonably be associated with aggregate emission, is not enough to adequately fit the $0-1$ peak near $625 \mathrm{~nm}$; the best fit requires a substantial increase in the relative amplitude of the $0-1$ band as well as the $0-2$ /aggregate band (Table 1 ). Thus, while some of the relative change in height of the 585 $\mathrm{nm}(0-0)$ and $625 \mathrm{~nm}(0-1)$ PL bands may be due to the presence of emissive aggregates, another phenomenon must be responsible for the bulk of the difference.

The other possibility to explain the changes in the fluorescence of various MEH-PPV films is that the PL of the exciton changes in different film environments. This idea is supported by experiments examining the behavior of the PL from conjugated polymer films with applied pressure. ${ }^{87,88}$ As the 

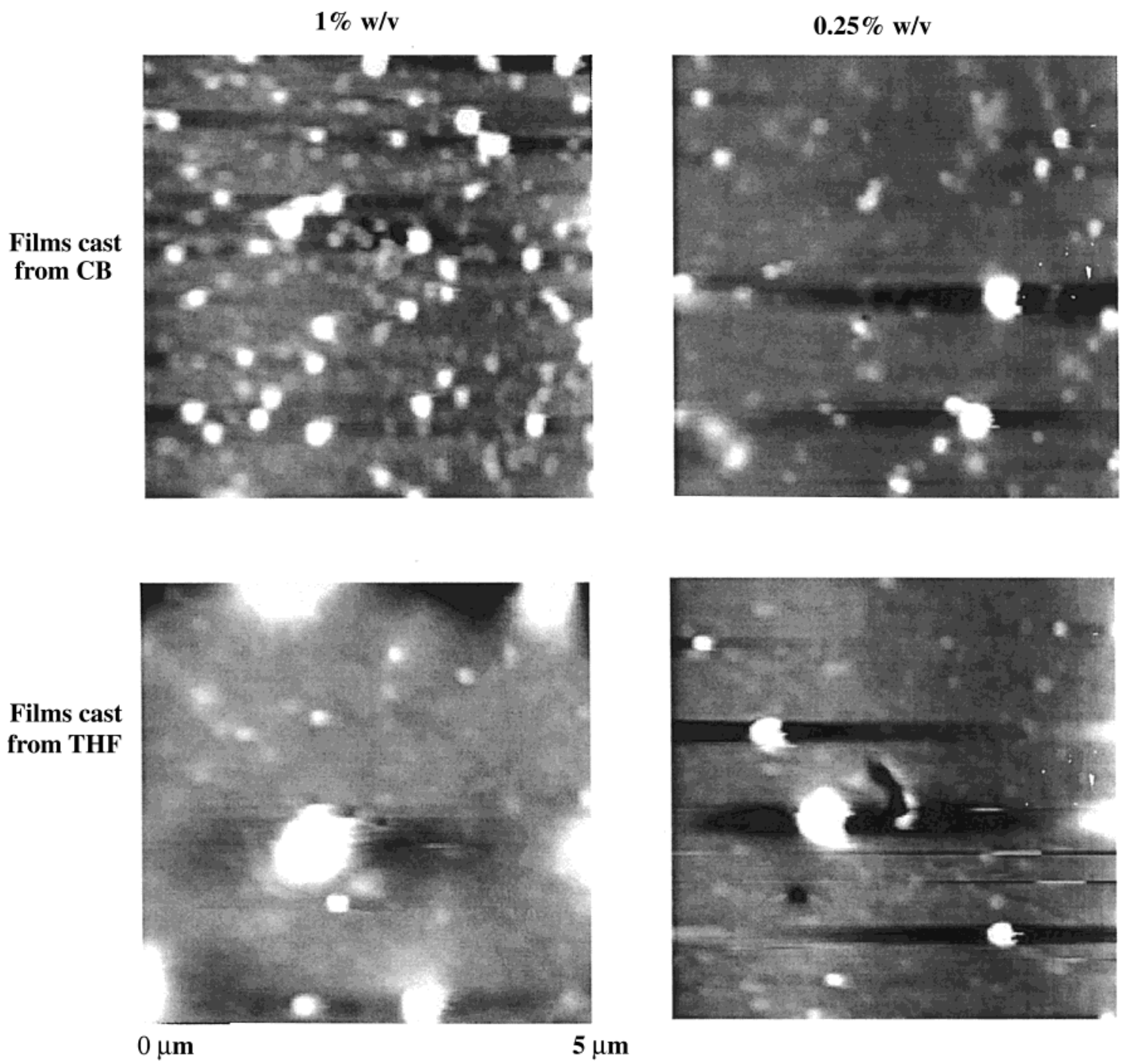

Figure 3. $5 \mu \mathrm{m} \times 5 \mu \mathrm{m}$ scanning force micrographs of MEH-PPV films cast from different solutions. Clockwise from upper left: $1.0 \% \mathrm{w} / \mathrm{v}$ in $\mathrm{CB}, 0.25 \% \mathrm{w} / \mathrm{v}$ in $\mathrm{CB}, 0.25 \% \mathrm{w} / \mathrm{v}$ in THF, $1.0 \% \mathrm{w} / \mathrm{v}$ in THF. The black-to-white color scale represents height differences of $100 \AA$. The dark horizontal streaks associated with some of the topographic features are artifacts of the horizontal scan direction.

pressure (and correspondingly the amount of interchain contact) is increased, the PL of MEH-PPV red-shifts, the height of the $0-1$ and $0-2$ peaks increase relative to the $0-0$ band, and the emission quantum yield drops-exactly the same changes we see with increasing degree of aggregation in the solution from which the film is cast. The vibronic structure in the PL of MEH-PPV remains visible at pressures up to $50 \mathrm{kbar}$, a result assigned to emission from a single intrachain species. ${ }^{88}$ The PL red-shift can be explained by the increasing polarizability of the environment, and the loss in quantum yield can be attributed to formation of an increased number of weakly emissive aggregates. The relative change in height of the emission peaks are then assigned to changes in vibronic coupling: ${ }^{88}$ both the Franck-Condon emission envelope and the number of sequence and combination bands underlying the main vibronic structure are likely to change as the interaction between a chromophore and its neighbors is altered. This idea of the exciton PL having environment-dependent vibronic structure is also supported by recent experiments using near-field scanning optical microscopy (NSOM) to examine single MEH-PPV chromophores diluted in an inert host polymer. ${ }^{89}$ The single chromophores clearly cannot be aggregated, but the emission spectrum from different chromophores shows the same type of variability in position and vibronic structure seen in Figure 1. Thus, we believe the changes in the emission spectrum of MEH-PPV films with increasing interchain interactions are predominantly the result of changes in the spectrum of the exciton, with increasing amounts of emissive interchain species playing an important but secondary role. The fact that the vibronic structure of the exciton changes with film morphology makes assignment of the fraction of the emission that comes from aggregates difficult. The relative increase in magnitude of the $660 \mathrm{~nm}$ band likely reflects a combination of a change in the relative strength of the $0-2$ emission from the exciton and an increase in the number of emissive aggregates.

All of the above arguments that the amount of aggregation in MEH-PPV films changes with the way in which the film is prepared are predicated on photophysical data. As of yet, we have provided no direct evidence that the morphology of the polymer films changes with increasing solution concentration or choice of solvent. X-ray diffraction work from several groups, however, indicates that the chain packing in conjugated polymer films, ${ }^{90}$ including $\mathrm{MEH}-\mathrm{PPV},{ }^{91,92}$ does strongly depend on the way in which the film was cast. In the next section, we offer direct evidence for the change in morphology of MEH-PPV films with casting conditions by performing scanning force microscopy on the same films whose PL spectra are presented in Figure 1(b). 
Annealed MEH-PPV

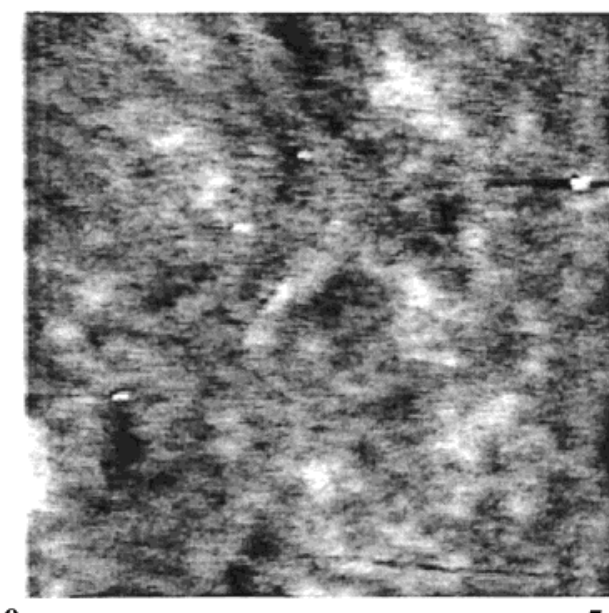

PS cast from THF

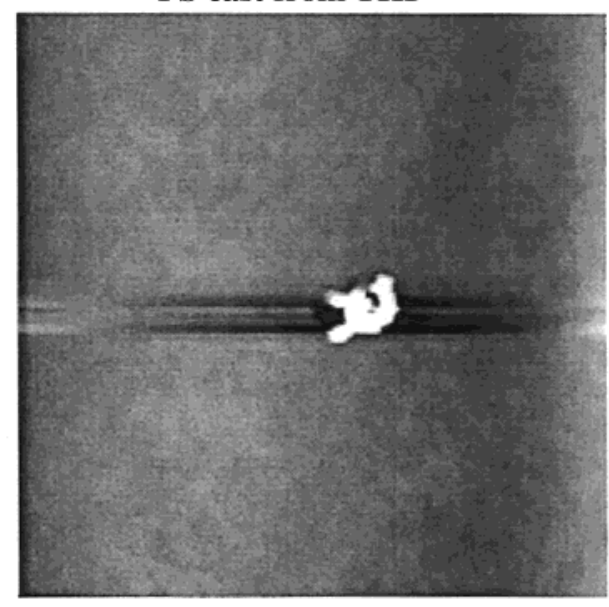

$\mathbf{0} \mu \mathbf{m}$

$5 \mu \mathbf{m}$

Figure 4. $5 \mu \mathrm{m} \times 5 \mu \mathrm{m}$ scanning force micrographs of an annealed MEH-PPV film cast from $1.0 \%$ CB solution (left) and a polystyrene (PS) film cast from THF (right). The black-to-white color scale represents height differences of $40 \AA$, less than half that in Figure 3 . The irregular object on the PS film is a dust particle (see text).

B. Morphology of MEH-PPV Films via Scanning Force Microscopy. Figure 3 shows scanning force micrographs of the MEH-PPV films cast from 1\% w/v CB solution (upper left), $0.25 \% \mathrm{w} / \mathrm{v} \mathrm{CB}$ solution (upper right), $1 \% \mathrm{THF}$ solution (lower left), and $0.25 \%$ THF solution (lower right). All of the scans show an area $5 \mu \mathrm{m}$ on a side; the color change from black to white represents a height difference of $\sim 100 \AA$. Scans taken on other regions of the same films are qualitatively similar to those presented in the figure. Figure 4 shows $5 \mu \mathrm{m} \times 5 \mu \mathrm{m}$ scanning force micrographs of the annealed MEH-PPV film cast from $1.0 \% \mathrm{w} / \mathrm{v}$ CB solution (left) and a reference film cast from a $1 \% \mathrm{w} / \mathrm{v}$ solution of $500,000 \mathrm{MW}$ polystyrene (PS) in THF (right). The color change for the images in Figure 4 represents a height scale of only $\sim 40 \AA$. The irregular object seen near the center of the PS image is a dust particle; other regions of the same film show no such features and are essentially flat on the scale presented. The dark horizontal streaks associated with some of the topographic features in both figures are artifacts of the horizontal scan direction used when producing these images.

Figure 3 makes it clear that the surface topography of the $\mathrm{MEH}-\mathrm{PPV}$ films changes significantly with the choice of casting solution. The film cast from $0.25 \%$ w/v CB solution shows several small circular topographic features, the largest of which are $\sim 200 \mathrm{~nm}$ in diameter and $\sim 10 \mathrm{~nm}$ high. When switching the casting solution concentration to $1.0 \% \mathrm{w} / \mathrm{v}$, the resulting MEH-PPV film has an increased number of these same features. The features are too regular in outline, have too flat an aspect ratio, and occur with too high a frequency to be assigned to dust particles or other impurities (cf. the dust particle in the PS reference film in Figure 4). Since the degree of aggregation in solution is quite a bit higher at higher concentrations, it makes sense to assign the features to regions where the MEH-PPV chains are highly aggregated. We have already argued that the open and stiff MEH-PPV in CB solution tend to aggregate together to promote $\pi$-electron interactions. ${ }^{28}$ Clumps of these aggregated chains from the solution that end up near the surface of the film would be too tangled to flatten during the spin-coating process, leading to the observed topographic features (we will discuss the dependence of the film topography on spin speed in a future publication ${ }^{83}$ ). We note that these features are far too large to be the photophysical aggregates discussed above; rather, we believe that they correspond to regions of highly tangled MEH-PPV chains. We expect that the entangled chains in these regions have a higher propensity for interchain contact and thus a higher number of the photophysical aggregate species relative to flatter regions.

The idea of tangled MEH-PPV chains surviving casting from solution to the film also fits with the surface topography of the films cast from THF. The chain conformation in THF solution is highly coiled; ${ }^{28}$ thus, the ability for multiple chains to snarl together is significantly reduced relative to $\mathrm{CB}$ solution. The tightly coiled chains that do tangle in THF solution likely do so by agglomeration of entire chain coils. Thus, the film cast from $0.25 \%$ THF solution shows fewer topographic features than that from the corresponding $\mathrm{CB}$ solution, and the features that are present in the THF-cast film are larger in both diameter and height. Increasing the concentration of the THF solution to $1.0 \%$ promotes aggregation by increasing agglomeration of the coiled chains, producing even larger topographic features in the resultant cast film without significantly increasing their number.

Figure 4 shows that annealing produces yet another change in the surface topography of MEH-PPV films. The annealed films are significantly flatter than any of the as-cast films shown in Figure 3 (note that the color height scale in Figure 4 is less than half that in Figure 3). This fits well with a picture in which heating above $T_{\mathrm{g}}$ allows the chains to freely flow and form low energy structures. Annealing allows the MEH-PPV chains to slowly untangle and increase their degree of $\pi$-electron contact, producing a film surface that is nearly flat. The small features present on the surface of the annealed film show that some memory of the initial tangled regions is retained, but that for the most part, the polymer chains are now packed with a different morphology. Overall, Figures 3 and 4 argue that changing the conditions under which the film is prepared can control the morphology of conjugated polymer films.

C. Effect of Film Morphology on Photochemical Damage in Conjugated Polymers. As mentioned in the Introduction, several groups have argued that photooxidation of conjugated polymer films can take place quite readily, even at relatively low excitation intensities. ${ }^{39,40,49,74,75}$ The photophysical signatures of oxidative damage are a reduced PL quantum yield and the appearance of an excited-state absorption in the spectral region where SE is expected to occur. One suggestion for reducing photodamage is to excite the polymer film through the transparent substrate. ${ }^{49,74}$ If the film is optically thick, the photoexcited 


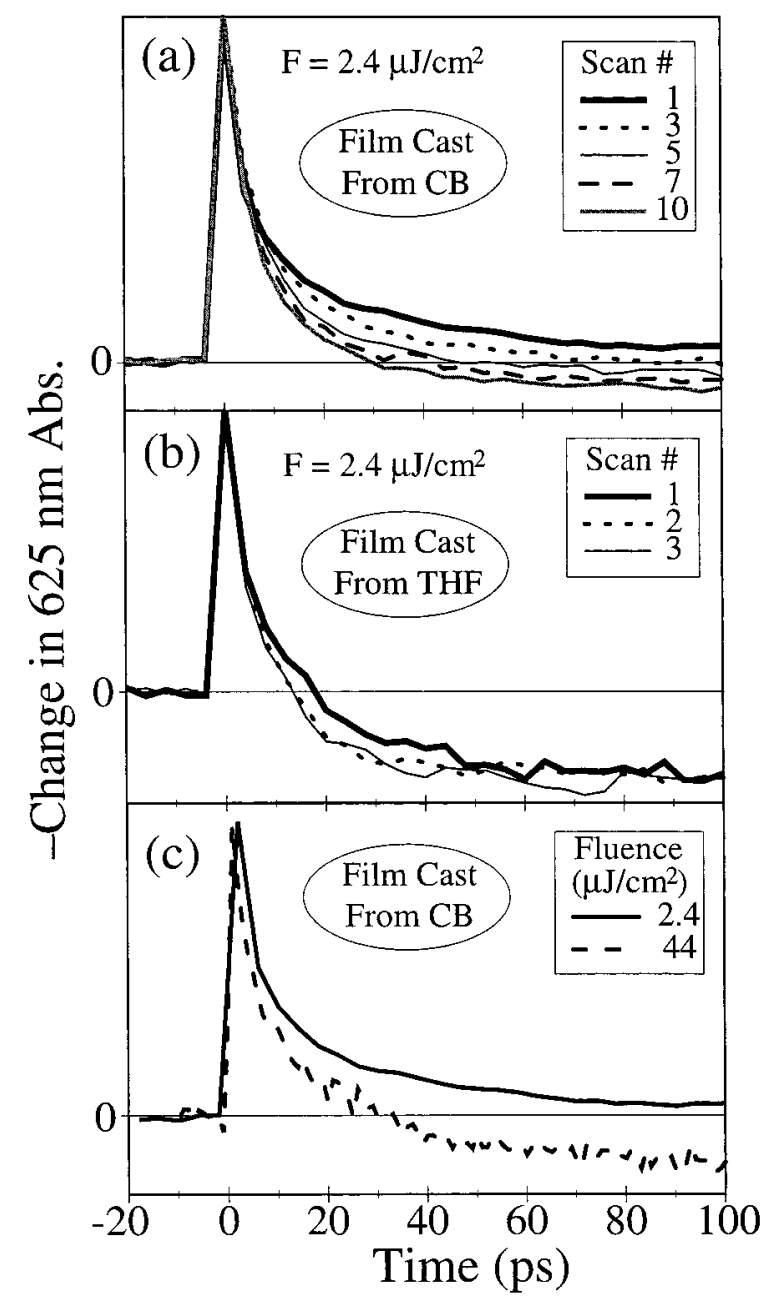

Figure 5. Degradation of the 625-nm SE dynamics in MEH-PPV films exposed to the air following excitation with femtosecond pulses at $485 \mathrm{~nm}$. Each pump-probe scan takes $\sim 1$ min to complete, and subsequent scans are taken moving the delay stage back and forth (see text for details). At a constant excitation fluence of $2.4 \mu \mathrm{J} / \mathrm{cm}^{2}$, the film cast from $1.0 \% \mathrm{w} / \mathrm{v}$ CB solution is monitored for 10 scans (a), while only the first three scans are shown for the film cast from $1.0 \%$ THF solution (b). The data in parts (a) and (b) of the figure have not been scaled. The scans at low $\left(2.4 \mu \mathrm{J} / \mathrm{cm}^{2}\right.$, solid curve) and high (44 $\mu \mathrm{J} / \mathrm{cm}^{2}$, dotted curve) excitation fluence on the CB-cast film shown in part (c) have been scaled to the same value at time zero.

region will have no contact with the outside environment since oxygen diffusion through the film is slow. Another way to reduce photooxidation is to keep the samples in the vacuum of a cryostat. We note, however, that irreversible sample damage assigned to oxidation has been observed even under vacuum. ${ }^{40}$

Figure 5 shows the results of femtosecond pump and probe experiments measuring the change in stimulated emission (SE) near the peak of the $0-1$ band at $625 \mathrm{~nm}$ following excitation near the MEH-PPV absorption maximum at $485 \mathrm{~nm}$. The data are plotted as the negative change in sample absorbance, so that an increase in probe intensity due to SE gain on the graph goes up and a loss of probe intensity due to excited-state absorption goes down. All of the experiments presented in this figure were performed on samples in air with the excitation light incident through the glass substrate. The stimulated emission dynamics shown in part (a) are for MEH-PPV films cast from $1.0 \% \mathrm{w} / \mathrm{v}$ solution in $\mathrm{CB}$; those in part (b) are for films of comparable thickness cast from $1.0 \% \mathrm{w} / \mathrm{v}$ solution in THF, both at constant excitation fluence $\left(F=2.4 \mu \mathrm{J} / \mathrm{cm}^{2}\right)$. A single pumpprobe scan was taken by averaging 300 laser shots $(0.3 \mathrm{~s})$ at each stage position and moving the translation stage to 100 stage positions. The total time to collect a single scan including stage movement is $\sim 1 \mathrm{~min}$. The stage was alternately translated backward and forward to collect multiple scans. Normally we would average the results of multiple scans together to improve the signal-to-noise. As is evident from the figure, however, the dynamics collected on subsequent scans are not reproducible.

Figure 5 ( $\mathrm{a}$ and $\mathrm{b}$ ) shows that the longer the sample has been exposed to the laser, the faster the decay of the SE and the greater the magnitude of the PA that appears at longer times. None of the data in Figure 5 ( $\mathrm{a}$ and $\mathrm{b}$ ) has been scaled, indicating the number of emissive species produced immediately after photoexcitation is the same for each scan. The change in dynamics from scan to scan is completely consistent with previous reports showing that cumulative photooxidative damage creates an absorbing species in the region where SE occurs. ${ }^{39-41}$ Similar experiments performed pumping from the front face of the sample or using films cast from $0.25 \%$ solution showed an even faster appearance of the absorbing species. This is consistent with the fact that the films cast from solutions of lower concentration are optically thin, resulting in a larger fraction of the photoexcited region of the sample near the polymer-air interface. For this reason, all of the pump-probe experiments shown in subsequent figures were performed only on films cast from $1 \% \mathrm{w} / \mathrm{v}$ solutions pumping through the substrate in a vacuum. Figure 5 (c) shows data collected on a fresh spot of the same film as part (a) at two different excitation fluences, $F=2.4 \mu \mathrm{J} / \mathrm{cm}^{2}$ (solid curve) and $F=44 \mu \mathrm{J} / \mathrm{cm}^{2}$ (dashed curve), scaled to the same maximum value for better comparison. Following the low fluence experiment, damage is evident after only a single scan at higher fluence. Once damaged, the low fluence result is not recovered and subsequent scans at low fluence are similar to the one obtained at high fluence. In the next section, we will show that similar experiments on samples in a vacuum show no PA at long times and have reproducible low fluence scans before and after high fluence excitation, verifying that exposure to air is indeed involved in the damage mechanism.

Perhaps the most interesting result in Figure 5 is that sample damage appears to occur more readily in $\mathrm{MEH}-\mathrm{PPV}$ films cast from THF than those cast from CB. Figure 5 (b) shows that the THF-cast film is damaged on the first scan; the equivalent amount of damage does not occur in the CB film at the same pump fluence until after 10 scans (Figure 5 (a)). We believe the difference in damage rate is directly related to the morphology of the MEH-PPV films. If the conformation of the polymer chains in solution is preserved into the film as suggested by Figure 3, then the MEH-PPV films cast from THF are comprised of tightly coiled polymer chains that would tend to pack roughly like spheres. The CB-cast films, on the other hand, would contain straighter polymer chains that tend to interpenetrate. The extra void space present between the chain coils in THF-cast films would allow oxygen to diffuse to photoexcited regions more readily than would the tight chain network in the CB-cast films, increasing the rate at which damage occurs.

The idea that changes in film morphology affect the rate at which MEH-PPV samples damage due to changes in oxygen mobility is also supported by recent NSOM experiments from DeAro and Buratto. ${ }^{93}$ In these experiments, MEH-PPV films in air were selectively excited either on top of one of the topographic features seen in Figure 3, or in one of the flat regions between the features. The total fluorescence intensity was monitored as a function of time. The emission intensity decreased following excitation due to photooxidation, but the 


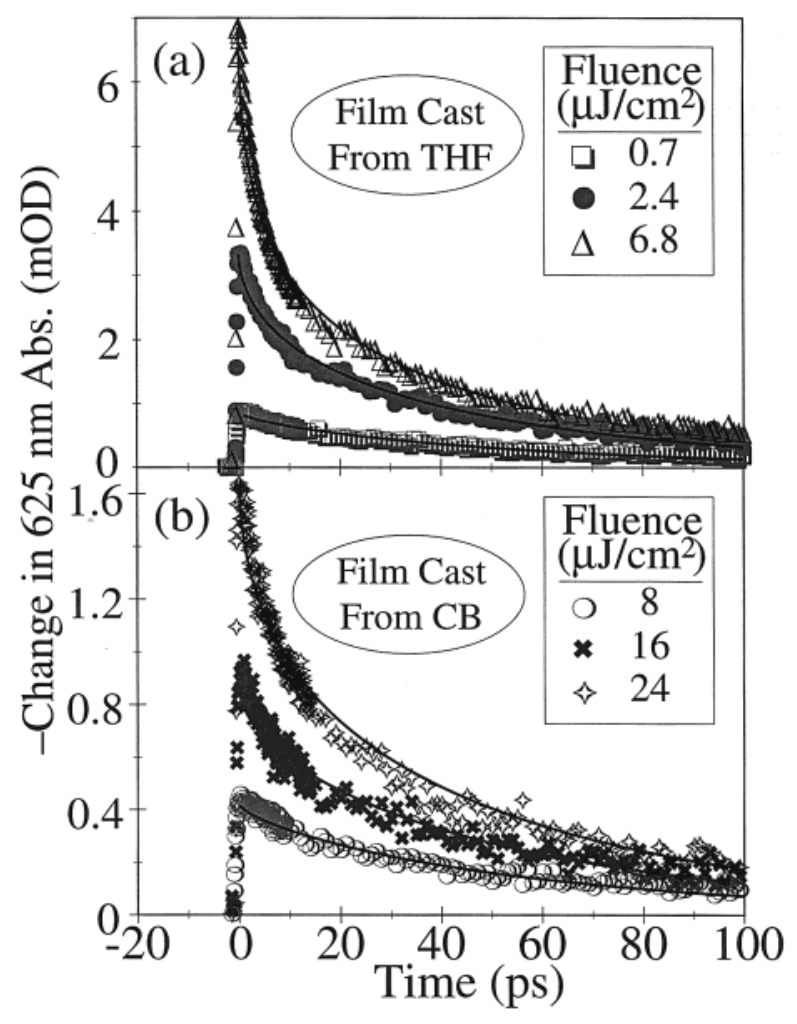

Figure 6. Effects of morphology on the intensity-dependence of the exciton dynamics in MEH-PPV films. (a) Pump 485/Probe $625 \mathrm{SE}$ scans of an MEH-PPV film cast from $1.0 \%$ w/v THF solution with an excitation fluence of $0.7 \mu \mathrm{J} / \mathrm{cm}^{2}$ (squares), $2.4 \mu \mathrm{J} / \mathrm{cm}^{2}$ (solid circles), and $6.8 \mu \mathrm{J} / \mathrm{cm}^{2}$ (triangles). (b) Pump 485/Probe $625 \mathrm{SE}$ scans of an $\mathrm{MEH}-\mathrm{PPV}$ film cast from $1.0 \% \mathrm{w} / \mathrm{v} \mathrm{CB}$ solution with an excitation fluence of $8 \mu \mathrm{J} / \mathrm{cm}^{2}$ (open circles), $16 \mu \mathrm{J} / \mathrm{cm}^{2}$ (crosses), and $24 \mu \mathrm{J} / \mathrm{cm}^{2}$ (diamonds). The solid curves show the best fit for the data from each film to eq 2 (see text for details).

rate of the decrease was slower for excitation at one of the topographic features than for excitation in the flat regions of the film. If the topographic features represent regions of highly tangled chains as argued above, then we would expect slower oxygen diffusion into these sites and thus slower oxidative damage relative to the more open chain conformation in the flat regions of the film.

Finally, we point out that the observation of SE in MEHPPV films cast from $\mathrm{CB}$ is at odds with the original results presented by Rothberg and co-workers who found PA but no SE. ${ }^{8}$ In subsection E below, we will show that with sufficiently low excitation intensity in a vacuum, both THF and CB-cast films of MEH-PPV show long-lived SE signals. The signal sizes reported in ref 8 suggest an excitation fluence more than 2 orders of magnitude higher than the high fluence results presented in Figure 5 (c). Thus, in agreement with the conclusions of others, ${ }^{39,49}$ we believe the lack of SE observed in ref 8 is not due to the presence of interchain species but instead is the result of photodamage from very high intensities. With this understanding of the nature of sample damage and its morphology dependence, in the next section we study how the intensity dependence of the photophysics of MEH-PPV depends on the degree of interchain interactions under conditions where oxidative damage does not occur.

D. Role of Film Morphology in Exciton-Exciton Annihilation. Figure 6 shows the intensity-dependent SE dynamics for MEH-PPV films cast from both (a) $1 \% \mathrm{w} / \mathrm{v}$ THF and (b) $1 \%$ w/v CB solutions. The data in Figure 6 (a) agree well with the essentially identical measurements reported by Dogariu et al. ${ }^{45}$ For these experiments, the excitation light passes through the substrate and the samples are kept under dynamic vacuum in a cryostat. Experimental reproducibility was verified by first running a scan at low excitation fluence, $\left(\leq 1 \mu \mathrm{J} / \mathrm{cm}^{2}\right.$ for THFcast films, $\leq 10 \mu \mathrm{J} / \mathrm{cm}^{2}$ for CB-cast films), then running the scan at the desired excitation fluence, and finally rerunning the scan at low excitation fluence. With all three scans taken using the same excitation spot, the before and after scans at low fluence were identical within the signal-to-noise. At excitation fluences $\geq 25 \mu \mathrm{J} / \mathrm{cm}^{2}$ for $\mathrm{MEH}-\mathrm{PPV}$ films cast from either solvent, however, we observe a turnover of the SE into a PA at long delay times. The magnitude of the time zero signal at these higher pump intensities scales sub-linearly with excitation fluence, and the before and after low-fluence scans do not agree, indicating that sample damage has occurred. The observation of sample damage at high fluences, even under dynamic vacuum, is in agreement with the observations of McBranch and co-workers who assigned the observed damage to photooxidation. ${ }^{40,42}$ In the next section, we will show that the annealing of MEH-PPV films produces a photophysical signature which is very much like that of photooxidation. Thus, we will assign the observed damage in a vacuum at high intensities to photoinduced annealing of the sample rather than photooxidation.

The intensity-dependent SE dynamics of the two films shown in Figure 6 are similar in two important ways. First, for the low excitation densities shown in the figure, the magnitude of the SE signals at time zero is linear with pump fluence, in agreement with other reports. ${ }^{44,45}$ This suggests that we are directly probing all of the emissive species created by the pump pulse and are not missing dynamics due to nonlinear interactions that occur faster than the resolution of our instrument. Second, the SE dynamics become increasingly faster at higher excitation intensity. These results are in accord with previous work on exciton-exciton (bimolecular) annihilation. ${ }^{40,41,43-45,48-50}$ The increasing SE decay rate with increasing excitation density also resembles that expected from amplified spontaneous emission. ${ }^{69,71,73}$ The data presented here, however, used excitation fluences nearly 2 orders of magnitude lower than those previously reported for the observation of line narrowing in MEH-PPV films. ${ }^{51,57}$ In addition, measurements of the PL using the same excitation pulses as for the pump-probe experiments showed no signs of line narrowing. Thus, we believe the intensity-dependent dynamics seen in Figure 6 are likely the result of bimolecular interactions.

In addition to their similarities, the SE decay results in Figure 6 also show two important distinctions between the $\mathrm{CB}$ and THF cast films. The first difference is that CB-cast films show a significantly smaller signal size than the THF-cast film at the same pump fluence. This can be explained partly by the fact that the CB-cast films have an optical density at the pump wavelength about half that in the THF-cast films (changing the solvent changes both the solution viscosity and evaporation rate, leading to films with different thicknesses), and partly by the fact that the CB-cast films have a lower total exciton quantum yield due to the higher fraction of aggregates. The second difference is that the same types of changes in SE dynamics that occur with a factor of 3 increase in pump fluence in the CB-cast film require an order of magnitude pump fluence increase in the THF-cast film. This is a key result of this paper: the rate at which E-EA occurs in MEH-PPV depends on the morphology of the film. This means that at high excitation densities, the local chain packing geometry determines the probability with which neighboring excitons can interact. This 
also could explain why the reported bimolecular recombination coefficients in different studies vary by nearly an order of magnitude. ${ }^{43,48,49,71}$ Studies on polymers with different side groups or on films prepared in different ways will have differing degrees of interchain interaction and, hence, different probabilities for bimolecular interaction.

Quantifying the difference in E-EA between MEH-PPV films cast from different solvents requires the use of a kinetic model. Previous studies of E-EA in conjugated polymers have used two different models to describe the effects of bimolecular recombination: ${ }^{40-45,48-50}$

$$
\begin{aligned}
& \frac{\mathrm{d} N(t)}{\mathrm{d} t}=-\frac{N(t)}{\tau}-\beta N^{2}(t) \\
& \frac{\mathrm{d} N(t)}{\mathrm{d} t}=-\frac{N(t)}{\tau}-\frac{\beta}{\sqrt{t}} N^{2}(t)
\end{aligned}
$$

where $N(t)$ is the time-dependent population density of emissive excitons (with the density of excitons at time zero, $N(0)$, directly proportional to the size of the SE gain signal), $\tau$ is the exciton lifetime, and $\beta$ is the bimolecular recombination coefficient. In the absence of the annihilation term or at low excitation densities, both models predict single exponential relaxation with the fluorescence lifetime; the quadratic term in both models represents interactions between excitons that allow for a faster decay of the population at high excitation densities. Both models assume that the measured SE transients are due only to excitons; interchain species are not taken into account. Using either model, a single choice of $\beta$ and $\tau$ should describe the exciton decay dynamics for all initial excitation densities. Thus, several pumpprobe transients with different excitation densities must be fit simultaneously to determine which model best describes the data.

The model represented by eq 1 assumes that excitonic diffusion maintains a spatially uniform carrier density at all times so that the annihilation rate depends only on the population density. The analytic solution to this model is given by

$$
N(t)=N(0)\left[\frac{\mathrm{e}^{-t / \tau}}{\tau+\tau \beta N(0)-\tau \beta N(0) \mathrm{e}^{-t / \tau}}\right]
$$

The $1 / \sqrt{ } t$ term in the model represented by eq 2 allows for the fact that excitons can interact at a distance through overlap of their delocalized wave functions at times too short for diffusion to play an important role. ${ }^{94}$ Equation 2 has no analytic solution and thus must be fit to the data numerically. While most workers have chosen either eq 2 or eq 3 to model their data, both McBranch and co-workers ${ }^{41}$ and Dogariu et al. ${ }^{45}$ have considered both models in the analysis of their results. McBranch and co-workers found that eq 2 provided a much more satisfactory fit to the data than did eq $3 .^{41}$ Dogariu et al. transformed their data in such a way as to allow the functional form of the timedependent coefficient in front of the quadratic term in the model to be determined without assuming the form of either eq 2 or eq 3. In agreement with McBranch and co-workers, they found that a $1 / \sqrt{ } t$ term like that in eq 2 provided the best fit to the experimental data. ${ }^{45}$

For the MEH-PPV SE data presented in Figure 6, we also fit the data to both eqs 2 and 3, and, in agreement with both Dogariu et al. ${ }^{45}$ and McBranch and co-workers, ${ }^{41}$ we find that eq 2 provides a much better fit to the data than eq 3 . The best fits with eq 3 are visibly quite different from the data and give a sum of squared errors that is larger by at least a factor of 3 than the best fits to eq 2 . The best fits to eq 2 for each film are shown in Figure 6 as the solid curves. For the THF-cast film, the best fit parameters to eq 2 are $\tau=57 \pm 8$ ps and $\beta=4.3$ $\times 10^{-20} \pm 1 \times 10^{-20} \mathrm{~cm}^{3} / \sqrt{ } \mathrm{ps}$. The CB-cast film, in contrast, has best fit parameters of $\tau=73 \pm 10 \mathrm{ps}$ and $\beta=3.5 \times 10^{-19}$ $\pm 8 \times 10^{-20} \mathrm{~cm}^{3} / \sqrt{ } \mathrm{ps}$. The interpretation of the fitting parameters is clear. The exciton lifetime $\tau$ for both films is close to the same within the error of the fit. This suggests that the emitting species in both films is the same with a similar PL lifetime in the absence of bimolecular interactions; the slightly larger lifetime in CB likely reflects a small component of longlived aggregate emission, as will be discussed further below. Much more striking is the fact that the bimolecular recombination coefficient is nearly an order of magnitude larger in the CB-cast film than in the THF-cast film. This is a direct indication that the probability for E-EA depends on the morphology of the conjugated polymer film; as expected, the higher degree of interchain interactions in the CB-cast films produces a much larger probability for excitonic annihilation. This is consistent with assumptions underlying eq 2: the degree of E-EA depends on the overlap of two neighboring excitonic wave functions, which in turn depends on the relative position of the adjacent conjugated polymer chromophores.

The large difference in bimolecular recombination coefficient with casting conditions also can be used to explain the observation of line narrowing in $\mathrm{MEH}-\mathrm{PPV}$ films cast from THF but not from CB. ${ }^{51,56}$ In films cast from THF, the probability for bimolecular interaction is low enough that amplified spontaneous emission can take place at high excitation densities before E-EA significantly reduces the exciton density. In films cast from $\mathrm{CB}$, on the other hand, the bimolecular recombination coefficient is high enough that E-EA quickly reduces the exciton density so that amplified spontaneous emission cannot occur. We note that the data of Figure 1 suggests that it is possible to reduce the interchain interactions by casting films from a solution with lower polymer concentration. Thus, with the right preparation conditions, it is possible that line narrowing could be observed from CB-cast films of $\mathrm{MEH}-\mathrm{PPV}$.

In the next section, we will return to our argument that a significant fraction of the absorbed pump photons at low excitation intensities produce weakly emissive aggregates. Our contention is that a higher fraction of aggregates is produced upon excitation of CB-cast films than of THF-cast films. Thus, for a given excitation fluence, the corresponding density of emissive excitons is lower in films cast from $\mathrm{CB}$ compared to films cast from THF. This means the relative values of $\beta$ for the two films quoted above may not be accurate because we do not know the absolute emission quantum yields, leaving an undetermined scaling factor in the fitting procedure relating the initial excitation density to the signal size for each film. Moreover, eqs 2 and 3 do not account for the presence of multiple emitting species or the possibility that excitons can change their emission cross-section and lifetime via energy transfer to aggregated sites. Due to all this complexity, we question the utility of simple bimolecular recombination models in providing anything more than a qualitative description of the photophysics in conjugated polymers. The fact that the SE decay rate increases with increasing pump fluence suggests that E-EA does occur, but the details are clearly much more involved than the simple physics suggested by the model of eq 2. Overall, the strongest conclusion from Figure 6 comes from the fact that similar changes in SE require a much larger change in pump fluence in THF- than CB-cast films: the rate at which E-EA 


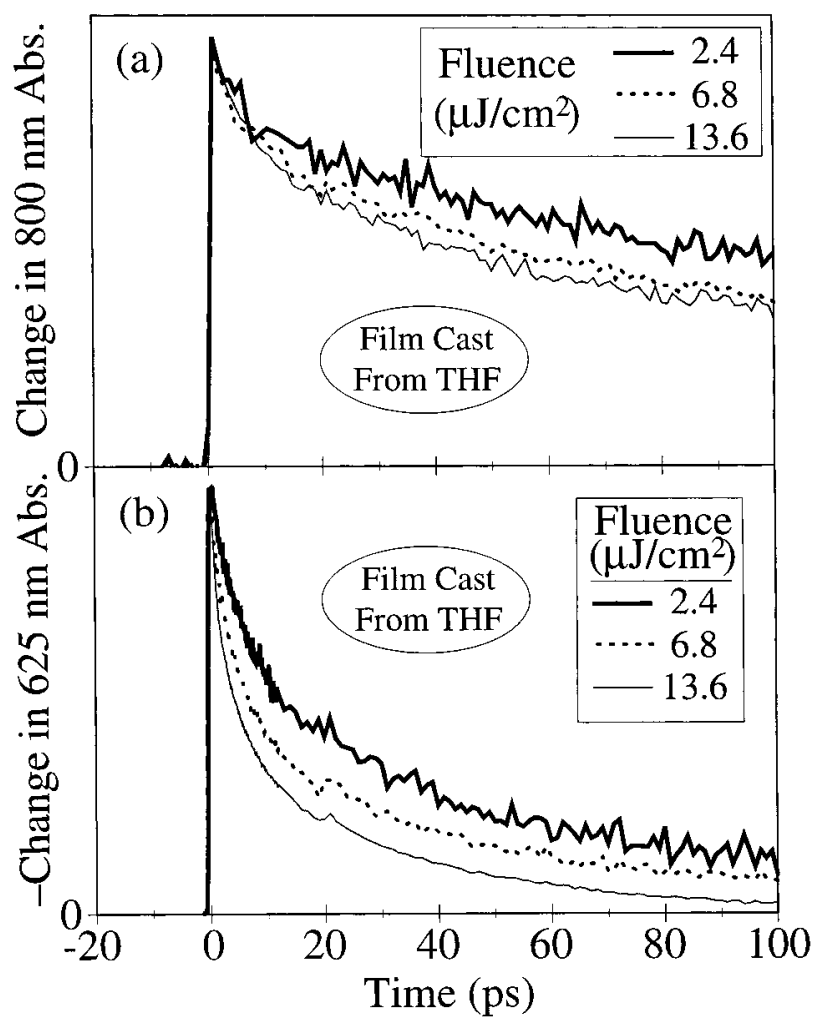

Figure 7. Comparison of the intensity-dependent 800-nm PA (a) and 625-nm SE (b) dynamics following 485-nm excitation for MEH-PPV films cast from $1.0 \% \mathrm{w} / \mathrm{v}$ THF solution with an excitation fluence of $2.4 \mu \mathrm{J} / \mathrm{cm}^{2}$ (heavy solid curves), $6.8 \mu \mathrm{J} / \mathrm{cm}^{2}$ (dotted curves), and 13.6 $\mu \mathrm{J} / \mathrm{cm}^{2}$ (thin solid curves). The SE data are plotted as the negative change in absorbance for ease of comparison, and both sets of data have been scaled to have the same signal at time zero.

occurs in MEH-PPV films is highly sensitive to the way in which the film was prepared.

We close this section by comparing the intensity dependence of the excited-state absorption of MEH-PPV films to that of the SE. Figure 7 (a) shows the $800 \mathrm{~nm}$ PA dynamics as a function of excitation fluence for an MEH-PPV film cast from THF. (Note that in this figure and in those below, the PA data is plotted as the change in absorbance rather than the negative of the change in absorbance used to plot the SE data.) The data in Figure 6 are all scaled to the same value at time zero for ease of comparison. The magnitude of the time zero PA scales linearly with excitation fluence in agreement with the recent work of McBranch and co-workers. ${ }^{44}$ The $625 \mathrm{~nm} \mathrm{SE}$ data at the same excitation fluences are shown in Figure 7 (b) (same data as in Figure 6 (a) but scaled to the same value at time zero). The change in PA dynamics at early times with intensity is in agreement with the ideas of McBranch and co-workers that a portion of the PA results from the byproducts of E-EA ${ }^{40-42}$ or of excitonic interactions with intrinsic defects. ${ }^{44}$ What is also clear from the figure, however, is that the PA and SE dynamics do not agree with each other, even at low intensities. This result is in accord with Dogariu et al. who also found differing PA and SE dynamics in MEH-PPV films cast from THF at low fluence, ${ }^{45}$ but it is not in agreement with identical SE and PA decays observed by McBranch and co-workers in an MEHPPV oligomer. ${ }^{40-42}$ (McBranch et al. do not compare the SE and PA dynamics in their more recent work on polymers. ${ }^{44}$ ) Thus, we turn in the next section to a more detailed study of the low intensity photophysics of MEH-PPV films, and we argue that the dissimilar dynamics are a direct result of the fact

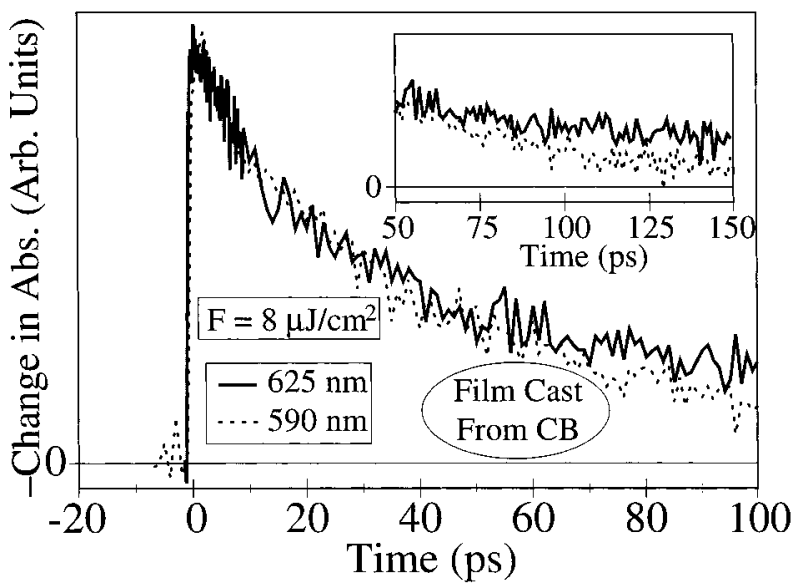

Figure 8. Comparison of the 625-nm SE (solid curve) and 590-nm SE (dotted curve) dynamics for an MEH-PPV film cast from a $1.0 \%$ $\mathrm{CB}$ solution following excitation at $485 \mathrm{~nm}$ with an excitation fluence of $8 \mu \mathrm{J} / \mathrm{cm}^{2}$. The inset shows the same SE transients on a longer time scale. The two transients in both the figure and inset have been scaled to the same value at time zero.

that photoexcitation of films cast from different solvents produces different fractions of excitons and weakly emissive aggregates.

E. Interchain Species at Low Intensity in MEH-PPV Films. Throughout this paper, we have argued that photoexcitation of MEH-PPV films produces both intrachain excitons and interchain aggregates, and that the relative fraction of aggregates produced depends on the film morphology. We have also argued that the aggregates have a weak emission on the red side of the PL, so the $625 \mathrm{~nm}$ SE dynamics transients presented above may have decay components from both excitons and aggregates. The PL excitation spectra presented above in Figure 2 and the work of Rothberg and co-workers, ${ }^{46,47}$ however, both suggest that MEH-PPV aggregates do not emit at the position of the $0-0$ band near $590 \mathrm{~nm}$. Thus, pump-probe SE transients at $590 \mathrm{~nm}$ should solely reflect the dynamics of the exciton. Since the exciton dynamics should be the same probed at either 590 or $625 \mathrm{~nm}$, any differences in the dynamics at the two wavelengths can be directly assigned to aggregate emission. Figure 8 makes exactly this comparison for the MEH-PPV film cast from $\mathrm{CB}$ : the solid curve shows the emission dynamics at $625 \mathrm{~nm}$ (same as in Figure 6 above), and the dashed curve shows the $590 \mathrm{~nm}$ emission dynamics at the same pump fluence. The two transients in the figure are scaled to the same signal size at time zero for better comparison. It is clear that the early time dynamics are identical, indicating that the majority of the emission at both wavelengths comes from a single species, presumably the exciton. At times longer than $60 \mathrm{ps,} \mathrm{however,}$ the data show a long-lived emissive tail at $625 \mathrm{~nm}$ that does not exist at $590 \mathrm{~nm}$ (Figure 8, inset). This must be due to emission from a second, long-lived species that emits at 625 $\mathrm{nm}$ but not at $590 \mathrm{~nm}$, undoubtedly the aggregate. We know of no way to explain the difference in emission dynamics at the two wavelengths without invoking the presence of two distinct emitting species. The absolute difference between the two curves is small, but because the difference persists for relatively long times, the tail still has a significant contribution to the integrated (steady-state) spectrum. The relative amount of aggregate emission can be computed by comparing the integrated area of the difference between the two transients to the total area under the $625 \mathrm{~nm}$ transient. The result suggests that $\sim 20 \%$ of the emission at $625 \mathrm{~nm}$ in MEH-PPV films cast from $1 \% \mathrm{w} / \mathrm{v} \mathrm{CB}$ solution is due to aggregates. 

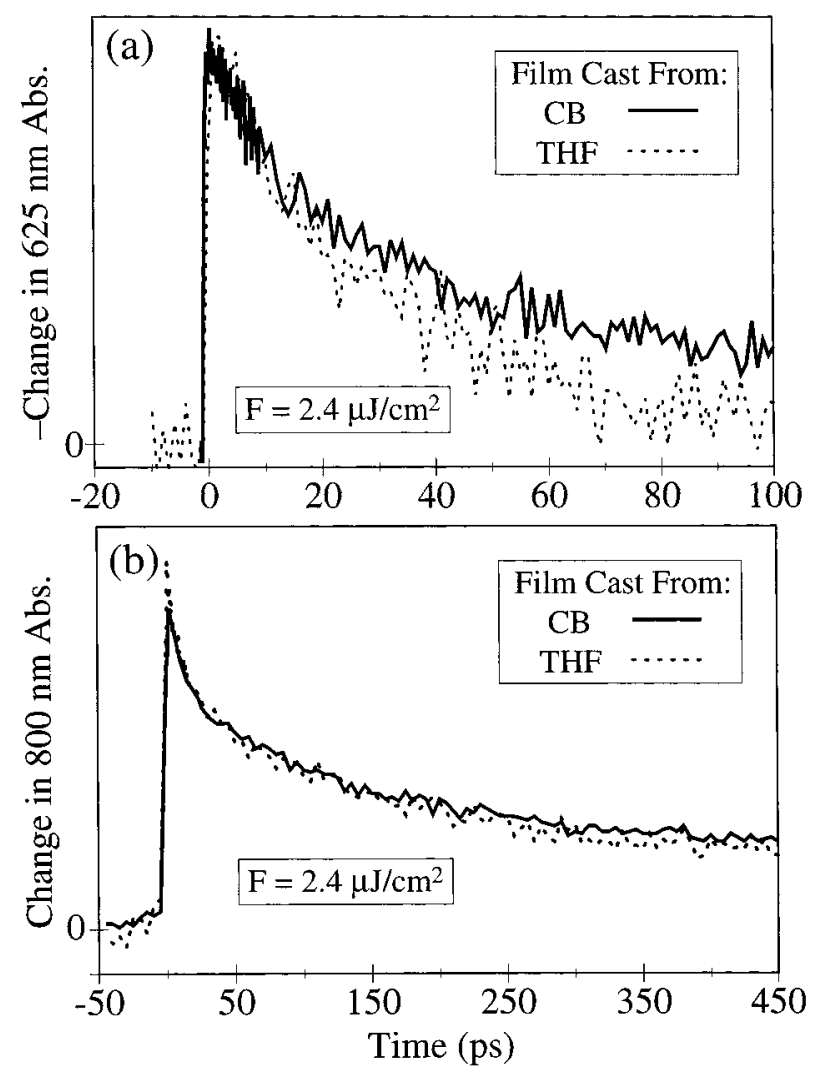

Figure 9. Comparison of the $625 \mathrm{~nm} \mathrm{SE} \mathrm{(a)} \mathrm{and} \mathrm{800-nm} \mathrm{PA} \mathrm{(b)}$ dynamics of MEH-PPV films cast from $1.0 \% \mathrm{w} / \mathrm{v}$ CB solution (solid curves) and $1.0 \% \mathrm{w} / \mathrm{v}$ THF solution (dotted curves) at a low excitation fluence of $2.4 \mu \mathrm{J} / \mathrm{cm}^{2}$. The SE traces in (a) have been scaled to the same maximum value at time zero; the PA traces in (b) have been scaled to have the same value at longer times (see text for details).

The data in Figure 1 presented above suggest that the ratio of aggregate to exciton emission should be higher in $\mathrm{MEH}-$ PPV films cast from CB than in films cast from THF. Figure 9 (a) shows the $625 \mathrm{~nm} \mathrm{SE}$ dynamics from the CB-cast (solid curve, same as in Figure 8) and THF-cast (dotted curve, same as in Figure 6) films at the same low pump fluence $(F=2.4$ $\mu \mathrm{J} / \mathrm{cm}^{2}$ ) and scaled to the same intensity at zero time. The dynamics agree well at early times, but at longer times the CBcast film shows an obvious long tail relative to the THF-cast film. Careful inspection of the data from the THF-cast film at long times (not shown), however, reveals a long-lived SE decay similar to that in Figure 8 but with a much smaller amplitude. Since the tail has larger amplitude in the CB-cast film, we believe that most of the difference in dynamics seen in Figure 9 (a) is due to the fact that THF-cast films have a smaller number of aggregates.

The dynamics of the PA in the CB and THF-cast MEHPPV films at low excitation fluence are shown in Figure 9 (b). Note that the time axis in this figure is compressed by a factor of 5 compared the data presented in Figures 6-8 and 9 (a), and that like Figure 7 (a), the data are plotted as the positive change in $800 \mathrm{~nm}$ absorbance. The data in this figure have been scaled so the two transients have the same change in absorption at long delay times. A long-lived, nonexponential tail that clearly does not have the same dynamics as the SE dominates the observed PA transients. Given that the photophysical signature of MEH-PPV aggregates in solution is a long-lived PA tail, ${ }^{28}$ the logical assignment for the long-lived PA species in Figure 9 (b) is the aggregate. This assignment is supported by the fact that the long-lived PA tail has qualitatively similar dynamics to the tails of the SE transients in Figures 8 and 9 (a); a

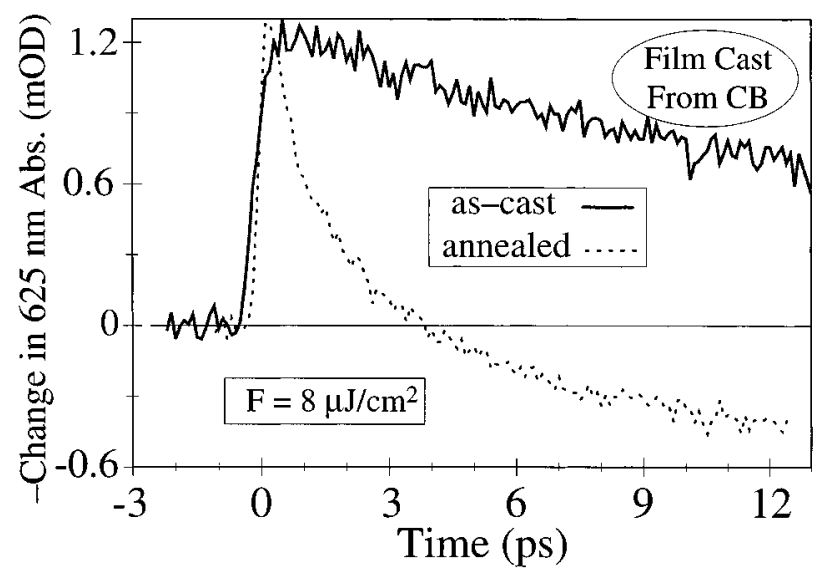

Figure 10. Comparison of the $625 \mathrm{~nm}$ SE dynamics for an MEHPPV film cast from $1.0 \% \mathrm{CB}$ solution (solid curve) and the same film after annealing (dotted curve).

quantitative comparison is difficult given the signal-to-noise in the SE experiments and the very low amplitude of the SE tails. In addition to aggregates, some of the PA could result from singlet or triplet ${ }^{95}$ excitons, dissociated excitons following encounters with intrinsic defects, or the byproducts of the small amount of E-EA that takes place at this excitation fluence. The fact that the tail comprises a significant fraction of the PA suggests either that many more interchain species than intrachain excitons are produced upon excitation, or that the 800-nm PA cross-sections of the interchain species are much larger than that of the exciton. The relatively weak intensity-dependence of the PA seen in Figure 7 (a) suggests that a large portion of the PA signal does not result from exciton dynamics because the change in exciton dynamics due to E-EA (cf. Figure 6) makes up a smaller part of the total PA signal. The PA signal in the long-lived tail is relatively unaffected by pump fluence, consistent with the assignment to aggregates.

Another striking feature of Figure 9 (b) is that the PA tails in the $\mathrm{CB}$ and THF-cast films are essentially identical. This means that whatever interchain species is responsible for the long-lived PA, the same species is created in both THF and $\mathrm{CB}$-cast films. The relative fraction of absorbing species is higher in CB than THF (as can be seen when scaling the two scans to the same amplitude at time zero instead of at long time), but the long-lived absorbing species produced in both films have the same dynamics. This supports the assignment of the longlived PA to aggregates; the identical decay time and higher relative PA amplitude in CB-cast films are consistent with all of the above data that suggest that aggregates form in both films but at a higher concentration in CB-cast films. Byproducts of E-EA are not expected to play a significant role in the PA transients of Figure 9 (b) because of the low excitation fluence, and there is no reason to expect that absorbing species produced by exciton dissociation at intrinsic defects would decay identically in the different films. All of the evidence presented in this paper consistently suggests that excitation of MEH-PPV films at low intensity produces a significant number of aggregates, in agreement with the recent conclusions of Rothberg and co-workers. ${ }^{46,47}$

Given that the size of the SE tail increases with increasing aggregation in the film, the ratio of aggregate to exciton emission should be even higher in the annealed films compared to the as-cast films. Figure 10 compares the 625-nm SE dynamics of the as-cast CB film (solid curve, same data as in Figures 8 and 9) and the same film after annealing (dotted curve) at a fluence low enough that there should not be significant E-EA $(8 \mu \mathrm{J} /$ 
$\mathrm{cm}^{2}$ ). Surprisingly, the annealed film does not show a large, long-lived emissive tail; instead, the SE decays into a net absorption after only $3 \mathrm{ps}$. This result is quite similar to that of Figure 5, where the SE quickly decayed into a PA following photooxidative damage of the sample. In the case of Figure 10, however, the film was annealed in a nitrogen environment and the excitation conditions (sample under dynamic vacuum, pumping through the substrate, low excitation fluence) are the same as for the data presented in Figures 6-9 where photooxidation does not occur. Thus, Figure 10 shows clearly that alteration of interchain interactions can produce defects with the same photophysical signatures as photooxidation. This is consistent with the idea of defect-mediated exciton dissociation; ${ }^{44}$ excitons take a few ps to migrate to the defect site, where they dissociate to produce an absorbing species (likely a charged polaron $^{43}$ ). This dissociation of excitons leads to the rapid decay of the SE, and the appearance of polarons causes the growth of the PA. The fact that such dynamics are observed in film samples that have never been exposed to oxygen indicates that these defects are intrinsic: defects can be formed by alteration of the interchain packing either via annealing or by photooxidation.

The possibility that intrinsic exciton dissociation produces a species which absorbs at the same wavelength as the emission has important implications for the photophysics of MEH-PPV. Pump-probe experiments measure a superposition of stimulated emission and excited-state absorption at a given wavelength. Thus, while most of the dynamics observed in pump-probe SE experiments reflect changes in the population of emissive species, some fraction of the measured dynamics may result from changes in the absorbance of dissociated excitons. In the experiments presented in Figures 6-9, the SE transients did not turn over into a PA tail at long delay times. At pump fluences higher than $\sim 25 \mu \mathrm{J} / \mathrm{cm}^{2}$, however, we did observe long time absorbing tails on the SE transients, indicating sample damage even in the vacuum of the cryostat. This is consistent with a mild annealing of the MEH-PPV films by the high intensity pump pulse, leading to an increased number of excitondissociating defects. It is worth noting that while a long PA tail indicates the presence of intrinsic defects, the converse is not true. The lack of observation of a PA tail at low excitation fluences does not necessarily mean that absorbing species do not play a role in the observed dynamics. Rather, the lack of a long time PA may indicate that absorbing species are present, but in low enough concentration (or with a low enough crosssection at the SE wavelengths) that the probe pulse experiences net gain rather than absorption. The long-lived emissive tail from the aggregates may also help mask small amounts of PA from dissociated excitons. We are presently constructing an apparatus that will allow us to simultaneously measure the SE and PL dynamics. Any differences observed will be a direct reflection of the presence of absorbing species modifying the dynamics in the pump-probe SE experiment.

The fact that aggregation in MEH-PPV films depends so sensitively on the casting conditions has important implications for the behavior of devices based on MEH-PPV films. Previous work has shown that changing the morphology of conjugated polymer films does have a significant effect on charge transport. ${ }^{90}$ Moreover, the quenching of emission due to the presence of aggregates or intrinsic defects is clearly detrimental to device performance. Thus, in the next section, we explore the behavior of MEH-PPV-based light-emitting diodes as the degree of aggregation and morphology of the active layer are changed by casting from different solvents.

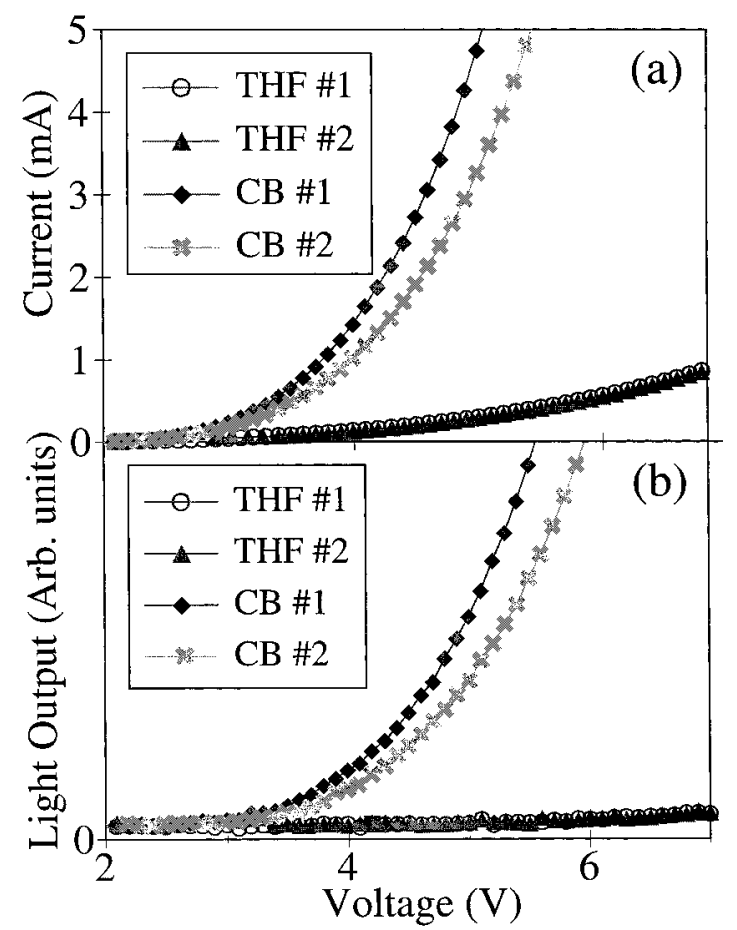

Figure 11. The dependence of current (a) and brightness (b) on applied voltage for ITO/PEDOT/MEH-PPV/Ca/Al sandwich-structure lightemitting diodes when the MEH-PPV layer is cast from $1.0 \% \mathrm{w} / \mathrm{v} \mathrm{THF}$ solution (circles, triangles) or $1.0 \% \mathrm{w} / \mathrm{v} \mathrm{CB}$ solution (diamonds, crosses).

F. Role of Film Morphology in MEH-PPV LightEmitting Diodes. Figure 11 shows current-voltage (a) and brightness - voltage (b) curves for ITO/PEDOT/MEH-PPV/Ca/ $\mathrm{Al}$ sandwich structure light-emitting diodes (LEDs). The diamonds and crosses show the data for two different devices constructed with the active layer cast from a $1 \% \mathrm{w} / \mathrm{v}$ solution of $\mathrm{MEH}-\mathrm{PPV}$ in $\mathrm{CB}$; the circles and triangles correspond to devices made with films cast from a $1 \%$ w/v MEH-PPV solution in THF. The data show clearly that the devices fabricated from the THF-cast films do not work as well as those based on CB-cast films. Figure 11 (a) demonstrates that the THF-cast devices have a higher turn-on voltage and lower working current than their CB-cast counterparts. Figure 11 (b) shows that the inability to get current into the THF-cast films results in a significantly lower light output: at $6 \mathrm{~V}$ bias, the emission from the THF-based devices is barely visible to the eye in a darkened room, while that from the CB-based devices is easily seen with the room lights on. To ensure that the differences in device performance were not artifactual, we fabricated and tested a series of devices from both CB- and THF-cast films. We found only minor differences in the behavior of devices that were prepared under identical conditions; the typical variation in performance is represented by the data for the two devices for each kind of film shown in Figure 11. Similar devices constructed without the PEDOT holetransport layer had higher turn-on voltages and lower working currents than those shown in Figure 11, but the qualitative behavior of lower current and lower light output when using THF-cast films remained the same.

The dramatic difference in the $\mathrm{I}-\mathrm{V}$ curves for devices based on MEH-PPV films cast from CB and THF in Figure 11 (a) directly reflects the different degrees of interchain interactions in the films. It is well known that spin-casting leads to polymer chains which lie primarily in the plane of the film. ${ }^{96}$ This means that it is unlikely that single polymer chains span the electrodes 


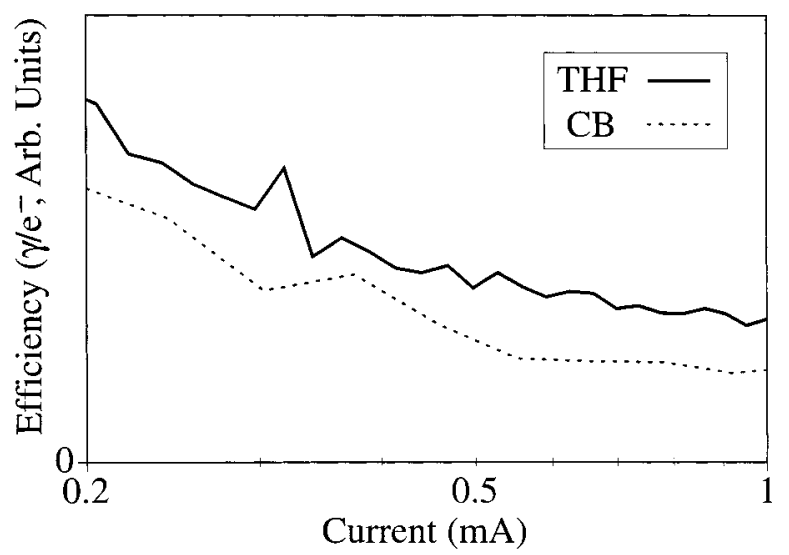

Figure 12. Efficiencies (in arbitrary units) for two of the MEH-PPV diodes whose brightness and current characteristics are shown in Figure 11 with the MEH-PPV film cast from $1.0 \% \mathrm{w} / \mathrm{v}$ THF solution (solid curve) or from $1.0 \% \mathrm{w} / \mathrm{v}$ CB solution (dashed curve).

in a sandwich-structure LED. Thus, current flow in conjugated polymer-based devices requires carrier transport between polymer chains, a process that is obviously sensitive to the degree of interchain interactions. For MEH-PPV in $\mathrm{CB}$, the open and straight polymer chains in solution form a film with large numbers of aggregates, facilitating charge transport between chains and allowing high current to flow between the electrodes. For MEH-PPV in THF, on the other hand, the tightly coiled chains in solution form a film with relatively weak interchain interactions. This means that there are few paths between the chains over which carriers can migrate through the film. As a result, it is difficult to both inject charge and carry current in the THF-cast films, leading to the observed higher turn-on voltage and generally poorer device performance.

The fact that THF-cast MEH-PPV-based devices perform more poorly than their CB-cast counterparts illustrates a fundamental tradeoff in maximizing the efficiency of conjugated polymer-based LEDs. Once the carriers recombine and the exciton is formed, the highest luminescence efficiency results from films with minimal interchain interactions. To form the emissive excitons by electrical injection, however, high carrier densities and mobilities are required, necessitating a large degree of interactions between the polymer chains in the device. This tradeoff is illustrated by the data in Figure 12, which shows the relative quantum efficiency (photons/electron) of the MEHPPV-based LEDs from Figure 11 as a function of current. The solid curve shows the efficiency of the device based on the THFcast film; the efficiency of the CB-cast film is shown as the dotted curve. The figure makes it clear that the efficiency of the device based on the THF-cast film is $~ 25 \%$ higher than that for the device fabricated from the CB-cast film. Thus, the few injected carriers that find their way to the center of the THF-cast film tend to recombine into excitons on single chains, producing luminescence with high efficiency. Many more carriers flow through the devices based on CB-cast films, but a significant fraction of those that recombine do so on aggregated sites leading to poorer overall luminescence efficiency.

This tradeoff between efficient charge transport and efficient luminescence with film morphology provides a prescription for optimization of conjugated polymer-based devices. For photovoltaic cells or photodiodes where charge transport is critical and luminescence is undesirable, casting the film from a solvent that maximizes interchain interactions can optimize the device. For polymer-based LEDs, the ideal situation would be to force the current through single polymer chains so that recombination can take place in an environment free of aggregation; recent work on isolated MEH-PPV chains in the channels of oriented mesoporous silica shows promise in this direction. ${ }^{36,97}$ For filmbased devices, the way in which interchain interactions should be optimized is less obvious. Finding the right balance between carrier transport and luminescence efficiency should enhance the performance of conjugated polymer devices. Another possibility to improve the efficiency would be to prepare a hetero-structure device based solely on MEH-PPV. By casting a layer from $\mathrm{CB}$ followed by a layer from THF and then another layer from $\mathrm{CB}$, the polymer in the device would have excellent current transport characteristics near the electrodes and a high luminescence efficiency in the center region where the carriers recombine.

We close this section by noting that the strong dependence of carrier transport on film morphology may also have implications for the mechanism of device failure. After operation for many hours, conjugated polymer-based LEDs develop "black spots" in which the luminescence is quenched and through which a pinhole short eventually forms. The scanning force micrographs in Figure 3 show that there are aggregated regions at the surface of MEH-PPV films. When these films are used in devices, it is likely that current injection takes place preferentially at the aggregated features on the surface. This means that most of the current through the device may flow through a few localized pathways in the film where the interchain overlap is the strongest. Since the current density through these aggregated paths is large, the polymer nearby is heated and probably anneals, and the annealed region grows with continued use. The emission from carriers that recombine in these annealed regions is strongly quenched, leading to dark spots where there is no luminescence. Eventually the resistance along the annealed pathways drops, leading to device failure when all of the current flows through a few nonemissive regions of the film.

\section{Conclusions}

Throughout this paper, we have argued that interchain species do exist in films of conjugated polymers and that the degree to which they form can be controlled by the way in which the film is prepared. In the Introduction, we pointed out that there has been a large amount of controversy in the literature concerning the role of interchain species in the photophysics of conjugated polymers. The samples in these studies, however, have consisted of oligomers, conjugated polymers with different side groups or molecular weights, conjugated polymer films prepared from solutions with different solvents or concentrations, and films spin-cast at different speeds. Since these different samples will have different degrees of interchain interaction, it is not surprising that so many authors have reached different conclusions. The data presented above show that interchain interactions change with both film morphology and excitation intensity, allowing resolution of many of the seemingly inconsistent results in the literature. Thus, we believe that morphology-dependent aggregation is a feature common to conjugated polymers as a class of materials.

While a majority of the change in shape of the PL spectra with increasing interchain interactions reflects changes in the vibronic structure of the exciton, some of the relative increase in the red edge of the PL is the result of aggregate emission. Since the aggregates have a much lower quantum yield for emission than the exciton, increasing the fraction of aggregates in the film lowers the overall PL quantum yield. Although absolute PL quantum yields of conjugated polymer films are difficult to measure, ${ }^{10,73}$ the data in Figure 1 provide enough information to determine reasonably accurate relative quantum 
yields. We can then approximate the total quantum yields of the different films using the measured relative yields by assuming that the quantum efficiency of the $1.0 \% \mathrm{w} / \mathrm{v} \mathrm{CB}$-cast film is the same $(\sim 12 \%)$ as that reported by Greenham et al. for $\mathrm{MEH}-\mathrm{PPV}$ films cast from the similar aromatic solvent xylene. ${ }^{10}$ These approximate quantum yields are summarized in Table 1. Not surprisingly, the quantum efficiency of the $\mathrm{MEH}-\mathrm{PPV}$ films drops in direct proportion to the extent of polymer aggregation in the solutions from which the films were cast. ${ }^{28}$ More importantly, the overall quantum yields presented in Table 1 provide a consistent relationship between the numbers of aggregates and excitons produced upon excitation of the different films. If we assume that the films cast from the low concentration solutions have a minimal degree of aggregation (as suggested by the PLE data in Figure 2), then the quantum yield for exciton emission must be $\sim 30 \%$, about the same as that observed in MEH-PPV solutions. ${ }^{28}$ Using the estimate of Rothberg and co-workers that half the excited species in $\mathrm{MEH}-$ PPV films cast from THF are aggregates, the overall quantum yield of 0.17 implies that the aggregates emit with $\sim 3 \%$ efficiency. A 3\% quantum yield is in accord with the ratio of the room-temperature PL and radiative lifetimes for the aggregate determined by Rothberg and co-workers. ${ }^{46,47}$ The emission from the $1.0 \% \mathrm{w} / \mathrm{v} \mathrm{CB}$-cast $\mathrm{MEH}-\mathrm{PPV}$ film can then be explained as a $\sim 1: 2$ exciton/aggregate mix, and the annealed film PL would result from a $\sim 15: 85$ ratio of excitons to aggregates. The 1:2 ratio in the CB-cast film implies that $\sim 20 \%$ of the total emission comes from aggregates, in excellent agreement with the magnitude of the long-time tail in the timeresolved SE data presented in Figure 8. The 85:15 mix of aggregates to excitons in the annealed film suggests that aggregates and excimers contribute roughly equally to the total PL seen in Figure 1. Of course, none of the above analysis takes into account that there may be nonemissive species that are also formed upon excitation (especially in the annealed films as implied by Figure 10). Still, the rough agreement of the approximate quantum yields in Table 1 with the estimates of Rothberg and co-workers ${ }^{46,47}$ provides strong support for the presence of significant numbers of morphology-dependent, weakly emissive interchain species in conjugated polymer films.

The existence of large numbers of aggregates in MEH-PPV films can also be used to resolve some of the controversy present in the literature. The dramatic change of the PL quantum yield with film preparation conditions is likely the reason for the large variation in the measured total PL efficiency for MEH-PPV reported by Greenham et al. ${ }^{10}$ The conclusions of Greenham et al. that there are not significant numbers of interchain species produced upon excitation of phenylene-vinylene polymers rely heavily on estimates of the radiative lifetime of the excition. ${ }^{10}$ Since absorption by aggregates comprises a fraction of the oscillator strength in conjugated polymer films, there is no simple way to directly estimate the exciton's radiative lifetime. The crudely determined numbers presented in Table 1 suggest that the emission quantum yield for excitons in MEH-PPV are at most a factor of $\sim 2$ higher than those reported by Greenham et al. We believe that the difference lies in errors calculating the radiative lifetime due to the presence of aggregates.

The presence of aggregates is also consistent with the results of published pump-probe experiments on conjugated polymer films. Our results are in good quantitative agreement with the MEH-PPV work of Dogariu et al. ${ }^{45}$ Moreover, the existence of aggregates is compatible with the "excitonic switch" work of Frolov et al. ${ }^{38}$ We believe that Frolov et al. chose a wavelength for the "dump" pulse in their experiments at which the excitons are strongly fluorescent but the aggregates do not emit (the equivalent of $590 \mathrm{~nm}$ for MEH-PPV as in Figure 8). This means that the only reduction in PA caused by the dump pulse would be due to the stimulated emission of excitons, exactly what Frolov et al. observed. ${ }^{38}$ Thus, if our conclusions for MEH-PPV hold for the alkoxy-substituted PPV used in the excitonic switch experiments, we would predict a different result only if a redder "dump" pulse were employed. We also note that Frolov et al. did observe a longer lived species that they assigned as resulting from triplet absorption; ${ }^{38}$ we believe that this PA possibly could be reassigned to aggregates. Finally, we point out that the solution-phase aggregation behavior of the polymer used by Frolov et al. is different from that of $\mathrm{MEH}-\mathrm{PPV},{ }^{31}$ so it is possible that the conjugated polymer films used for the experiments of ref 38 were cast in a way that produced relatively little aggregation.

The strong variation in interchain interactions with film morphology also plays an important role in the intensity dependence of the photophysics of conjugated polymers. Our results for $\mathrm{MEH}-\mathrm{PPV}$ are in good agreement with the polymer studies of McBranch and co-workers. ${ }^{44}$ It is likely that the oligomeric system studied by McBranch and co-workers ${ }^{40-42}$ has a morphology similar to that of MEH-PPV films cast from THF. Thus, the oligomeric films are easily damaged by photooxidation or by photoannealing and contain relatively few aggregates. The increased number of aggregates in conjugated polymer systems led to a much more congested photophysics because the PA of excitons, aggregates, the byproducts of E-EA (or exciton dissociation at intrinsic defects), and possibly triplets all overlap. Overall, we believe there is no inherent contradiction between the intensity-dependent results of McBranch and coworkers ${ }^{40-42,44}$ and the low intensity conclusions of Rothberg and co-workers ${ }^{46,47}$ because of the differences in film morphology.

Although the presence of significant numbers of interchain species implies that simple bimolecular recombination models miss much of the essential physics of conjugated polymer systems, we found that consistent fits to the SE data from the MEH-PPV films cast from both $\mathrm{CB}$ and THF could be obtained using eq 2. The bimolecular recombination coeffcient $\beta$ that we determined for CB-cast MEH-PPV films is nearly an order of magnitude higher than that in THF-cast films. This indicates that recombination is much more efficient in films with increased interchain interactions, so that the large variation in reported $\beta$ values can be explained by the different film preparation methods used in different studies. ${ }^{43,48,49,71}$ The fact that both the fluorescence quantum yield and $\beta$ vary so dramatically with interchain interactions also explains why the observation of line narrowing in conjugated polymers depends sensitively on how the films are made. ${ }^{51,54,55,72}$

Finally, the fact that the morphology and degree of interchain interactions in conjugated polymer films vary with casting conditions provides a prescription for optimizing polymer films for device applications. CB-cast MEH-PPV films with higher degrees of interchain interactions have lower overall luminescence quantum yields than THF-cast films, but they are better able to transmit charge carriers between chains. Annealed MEH-PPV films should provide even better charge transport characteristics at the expense of quenching nearly all of the emissive excitons. This means that the use of annealed films should significantly enhance the efficiency of conjugated polymer-based solar cells and photodiodes by allowing more efficient collection of charge through the polymer and reducing the losses from emissive recombination. The optimal film 
conditions for light-emitting devices require both that efficient charge transport moves carriers away from the electrodes and that recombination occurs in a region without significant interchain interactions. Given that the mobility of carriers along single polymer chains is high, ${ }^{98}$ the possibility of using single polymer chains for both charge transport and recombination could allow for a significant improvement in the efficiency of conjugated polymer-based devices. ${ }^{36}$

Acknowledgment. Acknowledgment is made to the donors of the Petroleum Research Fund, administered by the ACS (Grant. No. 32773-G6) and to the National Science Foundation (Grant No. DMR-9971842) for support of this work. B.J.S. is a Cottrell Scholar of Research Corporation and an Alfred P. Sloan Foundation Research Fellow. We thank Sonia Connolly of Sundown Arts for development of the computer-A/D interface software. We thank Chuck Knobler, Dylan Schiemann, and Hailan Guo for assistance with the scanning force microscopy measurements, and Yang Yang for assistance with preparation and characterization of the LEDs. We are also grateful to Fred Wudl and Roger Helgeson for the synthesis of the MEH-PPV, use of the fluorimeter for PL and PLE experiments, and to Kevin Sheran for assistance with the instrument for DSC. We thank Steve Buratto and Jessie DeAro for stimulating discussions and sharing NSOM results prior to publication, and Jennifer Sample and Jim Heath for use of the optical cryostat.

\section{References and Notes}

(1) Friend, R. H.; Gymer, R. W.; Holmes, A. B.; Burroughes, J. H.; Marks, R. N.; Taliani, C.; Bradley, D. D. C.; Dos Santos, D. A.; Brédas, J.-L.; Lögdlund, M.; Salaneck, W. R. Nature 1999, 397, 121.

(2) Braun, D.; Heeger, A. J. Appl. Phys. Lett. 1991, 58, 1982.

(3) Gustafsson, G.; Cao, Y.; Treacy, G. M.; Klavetter, F.; Colinari, N.; Heeger, A. J. Nature 1992, 357, 477.

(4) Bharathan, J.; Yang, Y. Appl. Phys. Lett. 1998, 72, 2660.

(5) Hebner, T. R.; Wu, C. C.; Marcy, D.; Lu, M. H.; Strum, J. C. Appl. Phys. Lett. 1998, 72, 519.

(6) Graupner, W.; Leising, G.; Lanzani, G.; Nisoli, M.; DeSilvestri, S.; Scherf, U. Chem. Phys. Lett. 1995, 246, 95.

(7) Blatchford, J. W.; Jessen, S. W.; Lin, L. B.; Lih, J. J.; Gustafson, T. L.; Epstein, A. J.; Fu, D. K.; Marsella, M. J.; Swager, T. M.; MacDiarmid, A. G.; Yamaguchi, S.; Hamaguchi, H. Phys. Rev. Lett. 1996, 76, 1513.

(8) Yan, M.; Rothberg, L. J.; Kwock, E. W.; Miller, T. M. Phys. Rev. Lett. 1995, 75, 1992.

(9) Schwartz, B. J.; Hide, F.; Andersson, M.; Heeger, A. J. Chem. Phys. Lett. 1997, 265, 327.

(10) Greenham, N. C.; Samuel, I. D. W.; Hayes, G. R.; Phillips, R. T.; Kessener, Y. A. R. R.; Moratti, S. C.; Holmes, A. B.; Friend, R. H. Chem. Phys. Lett. 1995, 241, 89.

(11) Yan, M.; Rothberg, L. J.; Papadimitrakopoulos, F.; Galvin, M. E.; Miller, T. M. Phys. Rev. Lett. 1994, 72, 1104.

(12) Jenekhe, S. A.; Osaheni, J. A. Science 1994, 265, 765.

(13) Samuel, I. D. W.; Rumbles, G.; Collison, C. J. Phys. Rev. B 1995, $52, \mathrm{R} 11753$

(14) Samuel, I. D. W.; Rumbles, G.; Collison, C. J.; Friend R. H.; Moratti, S. C.; Holmes, A. B. Synth. Met. 1997, 84, 497.

(15) Gebler, D. D.; Wang, Y. Z.; Fu, D. K.; Swager, M.; Epstein, A. J. J. Chem. Phys. 1998, 108, 7842.

(16) Osaheni J. A.; Jenekhe S. A. Macromol. 1994, 27, 739.

(17) Conwell, E. M. Synth. Met. 1997, 85, 995

(18) Conwell, E. M. Phys. Rev. B. 1998, 57, 14200.

(19) Wu, M. W.; Conwell, E. M. Phys. Rev. B 1997, 56, R10060.

(20) Lemmer, U.; Heun, S.; Mahrt, R. F.; Scherf, U.; Hopmeier, M.; Seigner, U.; Gobel, E. O.; Mahn, K.; Bassler, H. Chem. Phys. Lett. 1995, 240, 373.

(21) Pauck, T.; Hennig, R.; Perner, M.; Lemmer, U.; Siegner, U.; Mahrt, R. F.; Scherf, U.; Mullen, K., Bassler, H. Chem. Phys. Lett. 1995, 244, 171.

(22) Mahrt, R. F.; Pauck, T.; Lemmer, U.; Sieger, U.; Hopmeier, M.; Hennig, R.; Bassler, H.; Gobel, E. O.; Bolivar, P. H.; Wigmann, G.; Kurz, H.; Scherf, U.; Mullen, K. Phys. Rev. B 1996, 54, 1759.

(23) Blatchford, J. W.; Jessen, S. W.; Lin, L.-B.; Gustafsson, T. L.; Fu, D.-K.; Wang, H.-L.; Swager, T. M.; MacDiarmid, A. G.; Epstein, A. J. Phys. Rev. B 1996, 54, 9180.
(24) Blatchford, J. W.; Gustafson, T. L.; Epstein, A. J.; Vanden Bout, D. A.; Kerimo, J.; Higgins, D. A.; Barbara, P. F.; Fu, D.-K.; Swager, T. M.; MacDiarmid, A. G. Phys. Rev. B 1996, 54, R3683.

(25) Grell, M.; Bradley, D. D. C.; Long, X.; Chamberlain, T.; Inbasekaran, M.; Woo, E. P.; Soliman, M. Acta Polym. 1998, 49, 439.

(26) DeAro, J.; Weston, K. D.; Burratto, S. K.; Lemmer, U. Chem. Phys. Lett. 1997, 277, 532.

(27) DeAro, J. A.; Lemmer, U.; Moses, D.; Buratto, S. K Synth. Met. $1999,101,300$.

(28) Nguyen, T.-Q.; Doan, V.; Schwartz, B. J. J. Chem. Phys. 1999, 110,4068 .

(29) Samuel, I. D. W.; Rumbles, G.; Collison, C. J.; Moratti, S. C.; Holmes, A. B. Chem. Phys. 1998, 227, 75.

(30) Zheng, M.; Bai, F.; Zhu, D. J. Photochem. Photobiol. A 1998, 116, 143.

(31) Hsu, J. H.; Fann, W. S.; Tsao, P. H.; Chuang, K. R. J. Phys. Chem. A $\mathbf{1 9 9 9}, 103,2375$.

(32) Gelinck, G. H.; Warman, J. M.; Staring, E. G. J. J. Phys. Chem. 1996, 100, 5485 .

(33) Kersting, R.; Mollay, B.; Rusch, M.; Wenisch, J.; Leising, G.; Kaufmann, H. F. J. Chem. Phys. 1997, 106, 2850.

(34) Kersting, R.; Lemmer, U.; Mahrt, R. F.; Leo, K.; Kurz, H.; Bassler, H.; Gobel, E. O. Phys. Rev. Lett. 1993, 70, 3820.

(35) Warmuth, Ch.; Tortschanoff, A.; Brunner, K.; Mollay, B.; Kauffmann, H. F. J. Lumin. 1998, 76, 77, 498.

(36) Nguyen, T.-Q.; Wu, J.; Doan, V.; Tolbert, S. H.; Schwartz, B. J., submitted.

(37) Rothberg, L. J.; Yan, M.; Papadimitrakopoulos, F.; Galvin, M. E.; Kwock, E. W.; Miller, T. M. Synth. Met. 1996, 80, 41.

(38) Frolov, S. V.; Liess, M.; Lane, P. A.; Gellermann, W.; Vardeny, Z. V.; Ozaki, M.; Yoshino, K. Phys. Rev. Lett. 1997, 78, 4285.

(39) Vacar, D.; Dogariu, A.; Heeger, A. J. Chem. Phys. Lett. 1998, 290 58

(40) Klimov, V. I.; McBranch, D. W.; Barashkov, N. N.; Ferraris, J. P. Chem. Phys. Lett. 1997, 277, 109.

(41) Maniloff, E. S.; Klimov, V. I.; McBranch, D. W. Phys. Rev. B 1997, 756, 1876

(42) Klimov, V. I.; McBranch, D. W. Phys. Rev. B 1998, 58, 7654.

(43) Denton, G. J.; Tessler, N.; Stevens, M. A.; Friend, R. H. Synth. Met. 1999, 102, 1008.

(44) McBranch, D. W.; Kraabel, B.; Xu, S.; Kohlman, R. S.; Klimov, V. I.; Bradley, D. D. C.; Hsieh, B. R.; Rubner, M. Synth. Met. 1999, 101, 291.

(45) Dogariu, A.; Vacar, D.; Heeger, A. J. Phys. Rev. B 1998, 58, 10218. (46) Jakubiak, R.; Collison, C. J.; Wan, W. C.; Rothberg, L. J.; Hsieh, B. R. J. Phys. Chem. A 1999, 103, 2394.

(47) Jakubiak, R.; Rothberg, L. J.; Wan, W.; Hsieh, B. R. Synth. Met 1999, 101, 230.

(48) Kepler, R. G.; Valencia, V. S.; Jacobs, S. J.; McNamara, J. J. Synth. Met. 1996, 78, 227.

(49) Denton, G. J.; Tessler, N.; Harrison, N. T.; Friend, R. H. Phys Rev. Lett. 1997, 78, 733

(50) Zhang, J. Z.; Kreger, M. A.; Klaener, G.; Kreyenschmidt, M.; Miller, R. D.; Scott, J. C. Proc. SPIE 1997, 3145, 363.

(51) Hide, F.; Diaz-Garcia, M. A.; Schwartz, B. J.; Andersson, M. R.; Pei, Q.; Heeger, A. J. Science 1996, 273, 1833.

(52) Denton, G. J.; Tessler, N.; Stevens, M. A.; Friend, R. H. Adv. Mater. 1997, 9,547

(53) Zenz, C.; Graupner, W.; Tasch, S.; Leising, G.; Mullen, K.; Scherf, U. Appl. Phys. Lett. 1997, 71, 2566.

(54) Long, X.; Grell, M.; Malinowski, A.; Bradley, D. D. C. Opt. Mater. 1998, $9,70$.

(55) Long, X.; Malinowski, A.; Bradley, D. D. C.; Inbesekaran, M.; Woo, E. P. Chem. Phys. Lett. 1997, 272, 6.

(56) Hide, F.; Diaz-Garcia, M. A.; Schwartz, B. J.; Heeger, A. J. Synth. Met. 1997, 91, 35.

(57) Diaz-Garcia, M. A.; Hide, F.; Schwartz, B. J.; Andersson, M. R.; Pei, Q.; Heeger, A. J. Synth. Met. 1997, 84, 455.

(58) Frolov, S. V.; Gellermann, W.; Ozaki, M.; Yoshino K.; Vardeny, Z. V. Phys. Rev. Lett. 1997, 78, 729 .

(59) Frolov, S. V.; Gellermann, W.; Vardeny, Z. V.; Ozaki, M.; Yoshino, K. Synth. Met. 1997, 84, 471.

(60) Brouwer, H. J.; Krasnikov, V. V.; Hilberer, A.; Hadziioannou, G. Adv. Mater. 1997, 8, 935.

(61) Frolov, S. V.; Ozaki, M.; Gellermann, W.; Shkunov, M.; Yoshino, K.; Vardeny, Z. V. Synth. Met. 1997, 84, 473.

(62) Gelnick, G. H.; Warman, J. M.; Remmers, M.; Nieter, D. Chem. Phys. Lett. 1997, 265, 320

(63) Tessler, N.; Denton, G. J.; Friend, R. H. Nature 1996, 382, 695. (64) Diaz-Garcia, M. A.; Hide, F.; Schwartz, B. J.; McGehee, M. D.; Andersson, M. R.; Heeger, A. J. Appl. Phys. Lett. 1997, 70, 3191.

(65) Frolov, S. V.; Vardeny, Z. V.; Yoshino, K. Appl. Phys. Lett. 1998 72,1802 . 
(66) Frolov, S. V.; Shkunov, M.; Vardeny, Z. V.; Yoshino, K. Phys. Rev. B 1997, 56, R4363.

(67) McGehee, M. D.; Gupta, R.; Veenstra, S.; Miller, E. K.; DiazGarcia, M. A.; Heeger, A. J. Appl. Phys. Lett. 1998, 72, 1536.

(68) Kallinger, C.; Hilmer, M.; Haugeneder, A.; Perner, M. Adv. Mater. 1998, 10,920 .

(69) Cerullo, G.; Stagira, S.; Nisoli, M.; DeSilvestri, S. Phys. Rev. B 1998, 57, 12806.

(70) McGehee, M. D.; Gupta, R.; Veenstra, S.; Miller, E. K.; Heeger, A. J. Phys. Rev. B 1998, 58, 7035 .

(71) Haugeneder, A.; Hilmer, M.; Kallnger, C.; Perner, M.; Epstein, A. J. Appl. Phys. B 1998, 66, 389.

(72) Frolov, S. V.; Vardeny, Z. V.; Yoshino, K. Phys. Rev. B 1998, 57, 9141.

(73) Doan, V.; Tran, V.; Schwartz, B. J. Chem. Phys. Lett. 1998, 288 576.

(74) Harrison, N. T.; Hayes, G. R.; Phillips, R. T.; Friend, R. H. Phys. Rev. Lett. 1996, 77, 1881.

(75) Papadimitrakopoulos, F.; Konstandinidis, K.; Miller, T. M.; Opila, R.; Chandross, E. A.; Galvin, M. E. Chem. Mater. 1994, 6, 1563.

(76) Papadimitrakopoulos, F.; Yan, M.; Rothberg, L. J.; Katz, H. E.;

Chandross, E. A.; Galvin, M. E. Mol. Cryst. Liq. Cryst. 1994, 256, 663.

(77) Wudl, F.; Alleman, P. M.; Srdanov, G.; Ni, Z.; McBranch, D. ACS Symp. Ser. 1991, 455.

(78) Wudl, F.; Hoger, S., PCT Patent Application 1991, WO 94/20589.

(79) Fang, J.; Dennin, M.; Knobler, C. M.; Godovsky, Yu., K.;

Makarova, N. N.; Yokoyama, H. J. Phys. Chem. B 1997, 101, 3147.

(80) Cantor, D. R.; Schimmel, P. R. Biophysical Chemistry Part II; W. R. Freeman and Co.: San Francisco, 1980; p 454.
(81) Anderson, N. A. Ph.D. Thesis, University of Michigan, 2000.

(82) Holzer, W.; Penzkofer, A.; Gong, S.-H.; Bradley, D. D. C.; Long,

X.; Bleyer, A. Chem. Phys. 1997, 224, 315.

(83) Nguyen, T.-Q.; Schwartz, B. J., manuscript in preparation.

(84) See, e.g.: Hess, B. C.; Kanner, G. S.; Vardeny, Z. Phys. Rev. B 1994, 47, 1407.

(85) Shi, Y.; Liu, J.; Yang, Y., submitted.

(86) Ruhstaller, B.; Scott, J. C.; Brock, P. J.; Scherf, U.; Carter, S. A. Chem. Phys. Lett., in press.

(87) Webster, S.; Batchelder, D. N. Polymer 1996, 37, 4961.

(88) Tikhoplav, R. K.; Hess, B. C. Synth. Met. 1999, 101, 236.

(89) DeAro, J. A. Ph.D. Thesis, UC Santa Barbara, 1999.

(90) Zheng, W.; Angelopoulos, M.; Epstein, A. J.; MacDiarmid, A. G Macromol. 1997, 30, 7634.

(91) Weir, B. A.; Marseglia, E. A.; Chang, S. M.; Holmes, A. B. Synth. Met. 1999, 101, 154.

(92) Yang, C. Y.; Hide, F.; Diaz-Garcia, M. A.; Heeger, A. J. Polymer 1998, 39, 2299.

(93) DeAro, J. A.; Buratto, S. K. J. Phys. Chem. B, in press.

(94) Powell, R.; Soos, Z. J. Lumin. 1975, 11, 1.

(95) Monkman, A. P.; Burrows, H. D.; Migeul, M. da G.; Hamblett, I.; Navaratnam, S. Chem. Phys. Lett. 1999, 307, 303.

(96) McBranch, D.; Campbell, I. H.; Smith, D. L.; Ferraris, J. P. Appl. Phys. Lett. 1995, 66, 1175.

(97) Wu, J. J.; Gross, A. F.; Tolbert, S. H. J. Phys. Chem. B, 1999, 103, 2374.

(98) Hoofman, R. J. O. M.; deHaas, M. P.; Siebbeles, L. D. A.; Warman, J. M. Nature 1998, 392, 54. 